\title{
NBS Standard Reference Materials 1982-1983 Price List
}

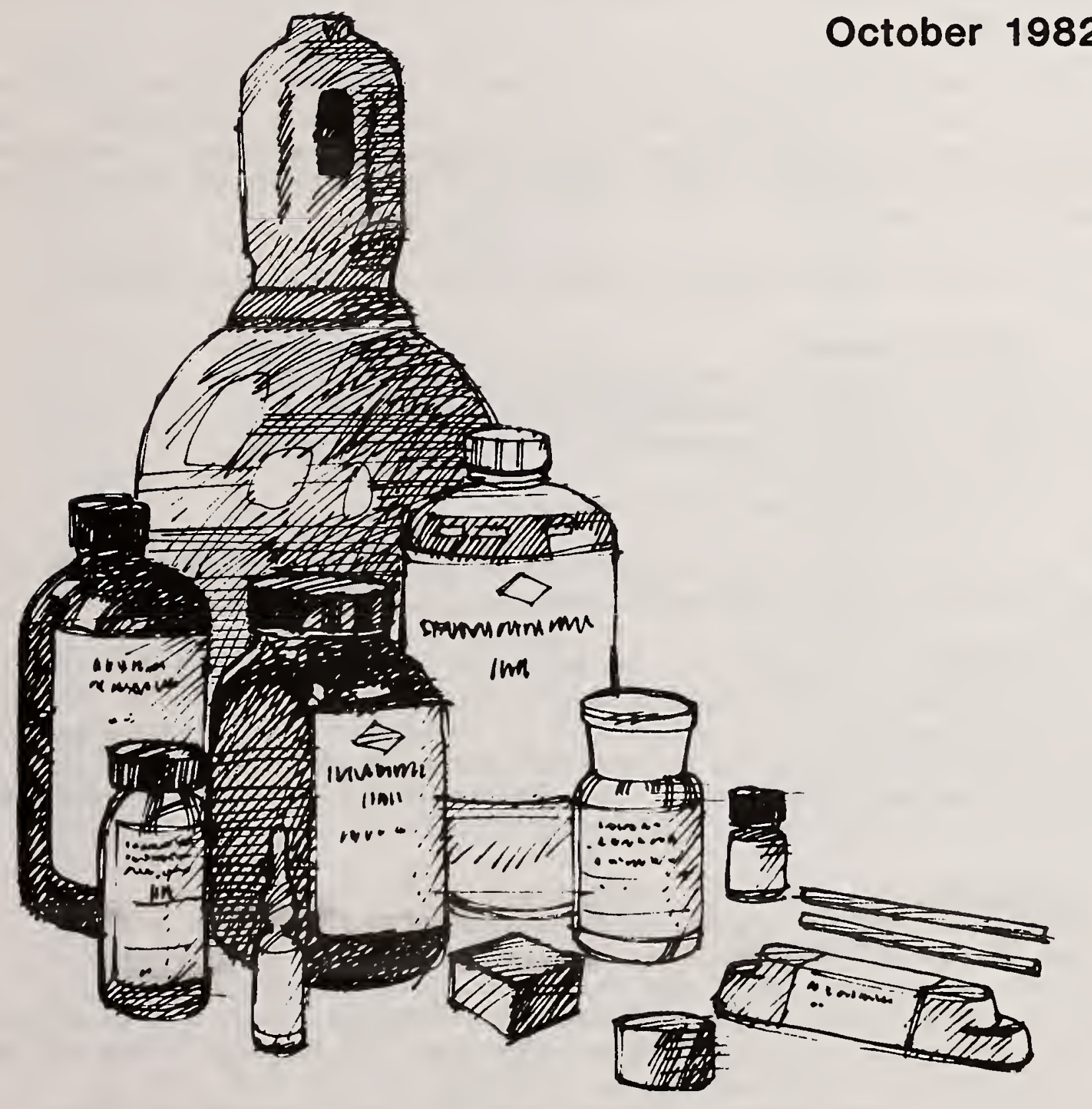

Office of Standard Reference Materials

U.S. DEPARTMENT OF COMMERCE

National Bureau of Standards

Washington, DC 20234

Telephone: 301-921-2045 
Orders should be addressed to the affice of Standard Reference Materals, Room B311, Chemistrr Building, National Bureau of Standards, Washington, U.C., 20234 (Telephone 301-921-2045). Drders should give the number of units, catalog number, and name of the standard requested. These materials are distributed onlr in the units 1 isted. Acceptance of an order does not implr acceptance of anr provision set farth in the order cantrarr to the policr, or resulations of the National Bureau of Standards or the the LIS Government.

Prices listed herein are subject to change without notice. Prices in effect at time of shipment will be billed to the purchaser. no discounts are given on NBS Standard Reference Materials. Parments not accampanring purichase orders are expected within 30 dars after receipt of invoices.

Parment of foreign orders mar be made br anr of the following:
a) UNESCO coupons,
b) banker's draft against U.S.A. toank,
c) bank to bank transfer to U.S.A. bank,
d) cash against documents,
e) sight draft, or
f) br International Moner Drder.

Proforma invaice service requires 3 to 4 weeks to process, and is furnished onlr to those customers requiring such service.

DUMESTIC SHIFMENTS of material (except for restricted categories) for the United States and Canada are shipped prepaid if the parcel does not exceed weight 1 imitations. For restricted categories and where the purchaser requests a special mode of shipment, the shipment will be sent collect. The Bureau does not prepar and add these shipping charges to the billing invoice.

FOREICN SHIPMENTS of materials are shipped by prepaid International Air Parcel Post, subject to size, weight, and categorr of material 1 imitatons. Anr other mode of shipment requested br a customer must be paid for br the custamer. Shipments excluded from Interiatianal Air Parcel Post are shipped Air Freight Dollect. Certain materials must be handled through an agent (shipping or brokerage firm) located in the U.S. as designated br the purchaser. These parcels will be packed for averseas shipment and forwarded express collect to the US firm designated as agent.

NOTE: All shipments are made in compliance with existing regulations pertaining to the material at time of shipment. 


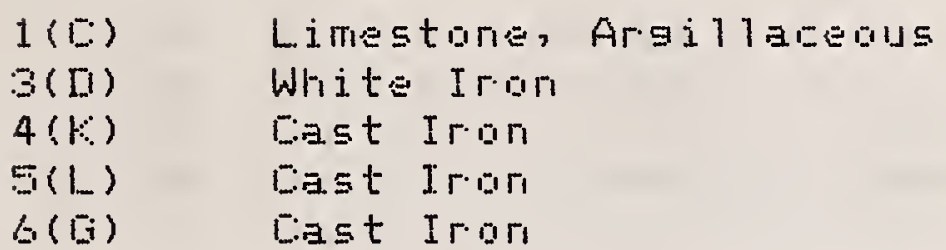

7(B) East Iron, (Hi-Fhos)

B(.1) Eartun St1, Eess 0.1L

1. $(H)$ Earban Et 1 , BDH O. $2 \mathrm{~L}$

$12(H)$ Garbon St 1 , EDH 0.45:

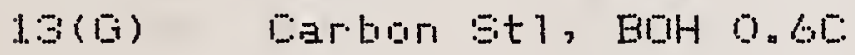

14(F) Earbar Et1, EDIH O. BE:

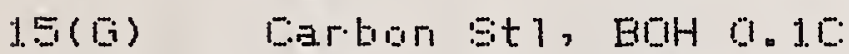

1G(E) Eartar St1, BDH 1.0L:

17 (E) SuErase, Palarimetric

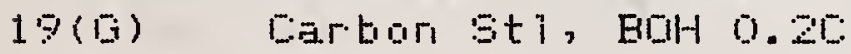

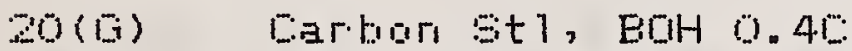

27 (F) Iron Gree, Sibler (Fowder)

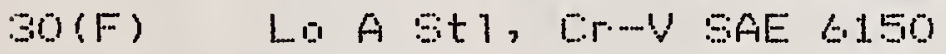

32 (E) LOA $E t 1$, Ni-Er SAE $\Xi 140$

$36(B)$

La $A S t 1, \quad[r 2-M a 1$

$37(E)$

$39(\mathrm{I})$

$40(H)$

4. (B)

$42(1 ;)$

$4:(H)$

$44(F)$

$45($ II)

$49(E)$

$50(E)$

$53(E)$

$54([1)$

$57(A)$

$5 E(A)$

$59(A)$

$64(\mathrm{C})$

$68(C)$

6 (B)

$70(A)$

71
Brass, Eheet

Eenzoil fird, Combustion

Sodium Dealate, Fedow

Dextrose, Polarimetric

Tin, Freezing Foint

Zine, Freezing Point

Al, Freezing Foint

Eu, Freezing Foint

Lead, Freezing Faint

To01 St1, W1.E-E.4-U1

Bearirg Metal, Lead-base

Eearing Metal, Tin-tase

Silican Metal

Ferrosjlicon, 75\%

Ferrosilicon, 50\%

Ferrobhromium, Hi Carbon

Ferromanganese, Hi Carbon

Eaurite (Arliansas)

Feldspar, Fotash

Caleium Molrtiate
509

$110=$

1509

$1.50 \div$

1509

150

1509

1509

$150=$

$1.50=$

1509

1.50

1509

609

$150 \Rightarrow$

$150 \div$

1009

150

1509

$150=$

$150=$

309

609

70 당

3509

3509

2009

$450 \div$

$600 \mathrm{~g}$

150 둔

1509

1.709

609

759

509

$1.00=$

150 9

60 잉

409

60
$\$ 72$

\$ 71

$\$ 71$

\$ 81

\$ 74

\$ 69

क 64

$+69$

$+69$

+ 69

$\$ 67$

\$ 69

क 69

+ 128

56

$\$ 67$

$\$ 90$

$+69$

म 69

\$ 69

$\$ 69$

\$ 67

+ 92

+ 59

$\$ 96$

+ 82

क 119

\$ 92

3 82

$+69$

* 69

$\$ 69$

क 73

\% 69

+ 81

(30

$\$ 94$

$+82$

$\$ 67$

\$ 62 
$72(15)$

$73(D)$

$7 E(A)$

$77(A)$

$7 B(A)$

$79(A)$

B1(A)

$83(\mathrm{DI})$

$84(-1)$

$B 5(\mathrm{~B})$

$\theta 7(A)$

$\theta \Xi(A)$

$B$

50

91

92

$98(A)$

$94(C)$

$97(A)$

$98(A)$

$9(A)$

$100(E)$

101(F)

$109(\mathrm{~A})$

105

106 (B)

$107(E)$

$113(\mathrm{~A})$

$11.4(N)$

$115(\mathrm{~A})$

$120(E)$

$121([1)$

$122(0)$

$123(0)$

125 (B)

$126(0)$

$127(\mathrm{E})$

$129(1)$

$131(\mathrm{C})$

$132(B)$
Lo A St1, SAE 4130

$S_{\text {. }} S_{\text {. }}$, Li.13 (SAE 420)

Burrit Refrart, A120:-40\%

Burrit Fefract, A1 $203-60 \%$

Burnt Fiefract, A1203-70\%

Fluorspar, Cinstoms Dirade Gilass Earid, High, Irari

Arseric Trioxide

Aloid Fotassium Frothalate

Aluminum Allor, Wrought

Aluminum-Silingr Allor

Limestore, Llolomitic

Lead Barium Lilass, Domp

Ferroptiosptiorous

Dpal [i]ass, Domp

Low Boror Gilass, Gomp

Borosilicate Lilass, Domp

Zri-tiase [ire-liastirig Allor

Elar, Flirit

Elar, Flastic

Feldspar, Soda

Lo A STL, Mriz SAE T1340

S. S., LR. 1 E-Ni 10 AISI $\Xi 041$

Btrrome Fefragtor\%

Lo A St1, Hi Si, E InI\%

La A St1, Dr-Mo-Al

Allor East Irori, Ni-lir-Mo

Zine Dre (Tri-State Gorre)

Coment Fineriess

Al 10 rast Irori, Eu-Ni-Lr

Frospriate fiol: (fouder)

S. S., TiO. 3 (SAE 321 )

East Iror, Ear Whes

$\Xi_{0} S_{\text {. }}$ N N 0.7 (SAE 347 )

LoA Steel, Si 3

$\mathrm{Hi}$ A Steel, Ni $\mathrm{Bt}$

Solder (Sr $40-F b G 0)$

St1, Fesul So.2 SAE 1112

Lo A Str, Si $([, S[\pi 1 \%)$

Too 1 St 1 , Mos-WG-Er.4-VZ
1509

$150 \mathrm{~g}$

759

759

759

1209

759

609

609

759

759

509

459

759

459

459

ea

$150=$

609

609

$40=$

1509

1009

609

1509

1509

$150 \mathrm{~g}$

$100=$

set (20)

$150=$

909

$150 \mathrm{~g}$

$150=$

1509

1509

$150=$

1509

$150=$

1509

1509
क 76

$\$ 69$

\$ 87

\$ 57

\$ 87

$\$ 74$

$\$ 60$

$\$ 1$

\$ 56

$\$ 69$

\$ 69

$\$ 67$

$\$ 58$

$\$ 62$

\$ 5

$\$ 59$

\$ 77

\$ 67

\$ 151

\$ 151

\$ 67

\$ 69

\$ 69

$\$ 59$

\$ 55

$\$ 69$

क 69

$\$ \quad 57$

\$ 89

\$ 69

$\$ 82$

क 64

\$ 73

\$ 64

\$ 77

$\$ \quad 64$

क 69

\$ 64

क 69

$\$ 64$ 
130 (E) S.5., Er $13-M 00.3-90.3$

$134(A)$ T001 St1, MoE-W2-Er.4-V1

136(D) Fotassium [ichramate

$1 \Xi 9$ (B) Etl, Er-Ni-MG, AISI. 8640

141 (D) Acetarilide, Mierachem

142 Anisie Acjid, Migrachem

143 (D) Drstine, Mierachem

1.49

Nicatinie feid, Micrache

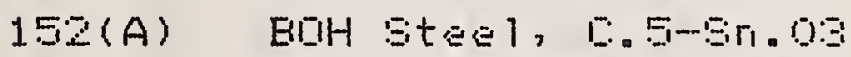

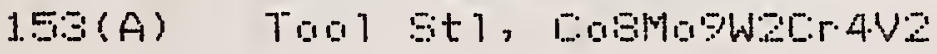

154(B) Titarium [iokide

15E La A St 1, Ero.5-W0.5

$158(A) \quad$ Eroras, Silican

$160(B) \quad$ S. S., AISI $\Xi 16$

169

Lo A St $1, \operatorname{Co.g-E.} 1.0$

165(A) [itass Bard

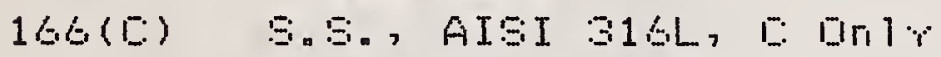

1.71

Magriesium-base Allor

176

Ti-tiase Allor, 5Al-2. ESn

1.78

Cartorn $5 t 1$, EOF O. 4L

179 Lo A Stl, High Si

100 Fluarspar, High Lirade

191 Lithium Gre (SFodumene)

192 Lithium ore (Fetalite)

193 Lithium Gre (Lefidalite)

1.5(E) Pat Hrdro Phthalate, PH

1EbI(E) Fat [itrodro Fhosphate, PH

JESIIL) [isod Hroro Flosphate, PH

$197(E)$

Eara\%, PH

1.96

Fot Hraragen Tartrate, $\mathrm{FH}$

189

Fot Tetrowalate, pH

1.91

192

Soldium Eicarlionate, FH

195

Eodium Earturiate, PH

Fat Nitrate, Fertilizer.

194

Ammonium [isho Fhos, Fert

195

196

196

199

200

Ferrosiljogr, HiFur (75\%)

Ferrochromium, Low

Silila Refr, 0.2\% A120

Silica Fefr, 0.5\% Al203

Fot Difr Fhosphate, Fert

1509

1.509

605

$150 \mathrm{~g}$

29

29

29

2

$150=$

1505

909

$150=$

1509

$1.50 \mathrm{~g}$

1009

759

1009

$100=$

100 의

150

$150=$

1209

$45 \div$

45 :

459

60

$30 \quad 9$

30 둥

30 9

609

659

30 잉

$30=$

90 ㄷ

90 9

$75=1$

100 드

$45 \div$

459

$0 \mathrm{a}$ $\pm \quad 6$

绾 69

\$ 92

年 64

$\$ \quad 57$

$\$ 57$

* 57

+ 53

56

क 69

\$ 89

+ 69

369

क 69

+ 81

\$ 69

\$ 55

年 69

569

辛 69

+ 69

+ 77

* 50

क 59

अ 5

$\$ 67$

\$ 72

+ 62

560

\$ 64.

\$24.

$\$ 65$

क

81

\$ 81

$\$ \quad 6$

\$ 89

क 59

क 59

\$ 80 
27á(A) Tungsten Eartide

277 Turasten Gorigentrate

279

$\because 1$.

293

30

30

31

30

39

304

30

39

30

39

$=40$

341

$342(\mathrm{~A})$

344

345

349

349

$350(A)$

$32(A)$

354

55

35

50

$30(A)$

$\because 6 \mathrm{j}$

36

263

$3: 4$

365

367

369

$270(E)$

(7) (C)

$372(1)$

$\because 9(F)$
Dtsidian Fig口:

LOA $\mathrm{At1}$, AETM A-213

$\because A S t 1$, AISI $B 620$

Zj.re Gingentrate, 45-Zn

Giaper Gre, Mill Hezds

Egpaer Gre, Mill Tails

Dofper Congentrate

Mo 7 rodenum concentrate

Grar Dast Iron, Des Grir

Earbu St o.tE, E arir

Gartion St $1.1 \mathrm{E}, \mathrm{E}$ an $r$

White Gast Iron, Es:

S. S. SAE OOBE

Ferraniatium

[IuEtile [ron

Nodular Irour

Hi A Stl, Mo peri hard.

Hi. A St1, Gu Fet hard.

Hi Temp Alla\%, A2BG

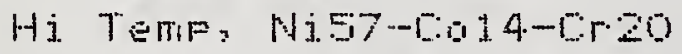

Benzais AEid, Frimar

Wra. 11 gred Ti. 20 Frm $H$

ingl 1 Gred Ti, $0.02 \% H$

Hhal lored Ti, g091. PFm 口 Hire 11 Gred Zr, H19,N49 FPM

Brat ared $2 \%, H 107$, N2GPFm

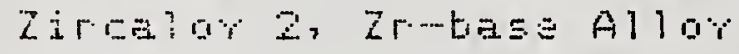

L. A $\mathrm{At}$, AISI. 4340

La A ETI, AISI 94517

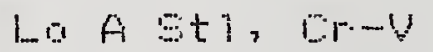

L. A St], Hi [ Had

[E] Etrotitj. Iror

S. S. ATSI 44G

Eteel, figi 1.21

Zirie Quide, Fut. Comp.

Sulfur, Fut. Comp.

Stearie AÉd, Fut. Comp.

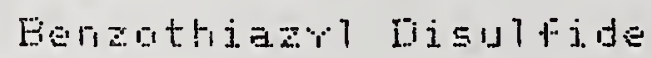

759
$100^{9}$
359
1509
1509

$100=$

1009

1009

509

359

$150 \quad \exists$

300 aㅗ

3009

1.509

1509

1009

1509

1509

1509

1509

1509

1.50 5

$\$ 09$

209

209

roid

$10 \doteq$ wire

10 g wire

1009

1.50

$150=$

1. 50

1509

1509

1509

1. $5(0)$

$81: 3$

$61: 9$

$3.2 \quad 1: 9$

$\therefore \quad 1:=$
D Eis

\$1.5

\$ 144

\$ 64

$\$ \quad 64$

$\$ \quad 57$

$\$ 2$

$\$ \quad 92$

韦

\$ 52

+ 67

$\$ 62$

\$ 62

\$ 67

\$ 81

क 89

$+69$

\$ 72

$\$ 69$

$\$ 69$

$\$ \quad 6$

क 69

* 105

क

\$ $\quad$ *

\$ 91

71

$\$ 75$

106

\$ 67

\$ 67

$\$ \quad 67$

* 67

\$ 67

\$ $6:$

(4) 6

\$ 97

\$ 75

\$ 71

古 77

* MATEFIAL IN PFEFAFATIDN 
SFIM

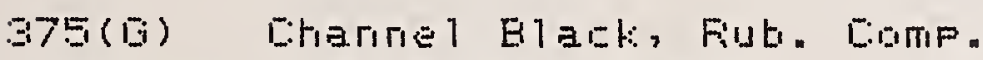

379 (E)

$302(A)$

$383(A)$

$394([1)$

$3 E G(H)$

$39 B(1)$

$3 \%$

314

395

396

$3 \%$

39

400

$404(A)$

$405 i(A)$

$407(A)$

$40 B(A)$

$40 \%$ (B)

$41:$

414

$4.7(A)$

$41 \Xi(A)$

$420(A)$

427

486

$4: 7$

436

489

4.40

441.

442

443

4.4 .4

445

4.46

447

4.98

449

4.50

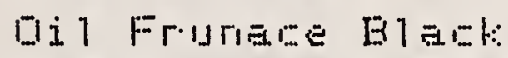

Lias Furrage Elagl:

Meroaptoberzathiazole

Siantoure, Fiut. Gimp.

Strrene Butadjere Fubtuer.

Eutr1 Fiubtier.

Una.11 ared [u ([.] O)

Una 11 ored [us ([DIJ I)

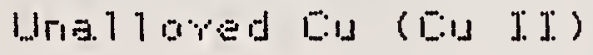

Hra 11 ared lou (LU III)

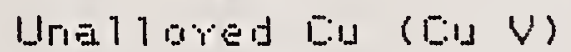

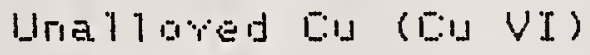

Uria 11 ard [D.] ([DU VIJ)

Gteel, Basia ElegtriE

Lo A stes 1, Mri 1. 9

La A Etee 1, Dr-V

La A Gtezl, Lir-Ni

Lo A Etes1, Ni 3

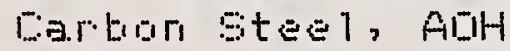

Lo A Etee1, Er-Ma

Larbun Stee1, EIDH O. 4.

Lo A St1, I. MM (SAE 4150)

Ingot I.r. nir

LDA St1, EAE4150(E DIIY)

$70015 t 1,515-M a-W 10$

Tou $5 t 1$, Cre-Ma-WS-Los

Tö

Tog St1, Ma H-S AISI MSG

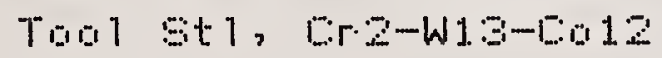

Tual Et1, WH-E AISI T1

E. E, Er $16-N i 10$

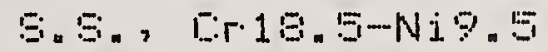

$5.5 .$, Lr20.5-Ni10

S. S. AIET 410

E. E, AIEI

$\Xi_{n} \Xi_{n}$, AISI 309

S.5., Algi. 403

S.5., L.5.5-Ni6.5

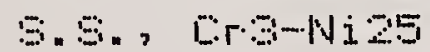

UNIT DF ISEUUE

FFICE

$29 \quad$ 1: 9

202

20 1:

32 1: 5

$3.21: 3$

ㄴ. 2 노

\$ 116

\$ 101

\$ 122

\$ 167

$\$ 4 \quad \therefore 5$

$34 \quad \mathrm{k}: 9$

505

501

E10)

$\$ 104$

\$211

+ $1: 2$

+ $1: 1$

\$ $1: 1$

509

- $1: 1$

50

509

505

r.01

\$ $1: 31$

$\$ 1: 1$

1. 131

古 64

rod

rog

rod

rod

rod

(4) 64

* 64

क 64

* $\quad 64$

\$ 64

r.

rogd

roget

r.g日

rog

\$ 64

\$ 64

范 64

\$ 64

\& 64

r.

rod

rog

r.at

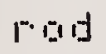

472

\$72

$\$ 72$

\$ 72

\$ 72

con

rad

r.ad

rod

rod

+ 72

\$ 72

\$ 72

\$ 72

\$ 72

1000

rong

rod

rod

roid
\$2
+72
$\$ 72$
$\$ \quad 72$
$\$ 72$ 


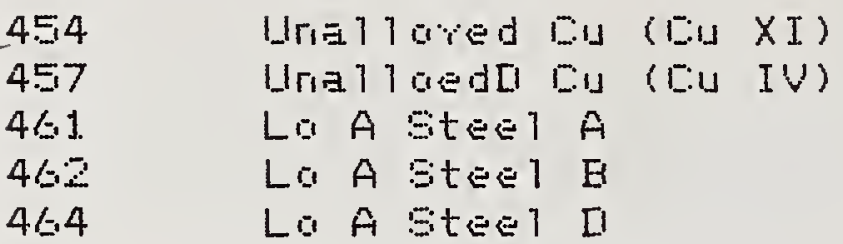

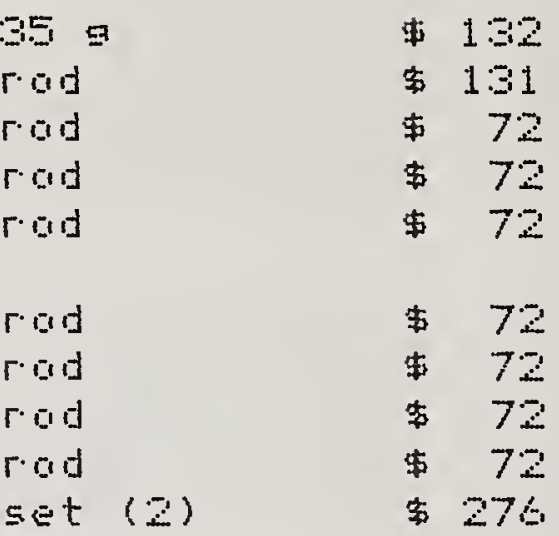

474 Afi Gr Dptical Linewidth 475 AF Er Getical Lirewidth $479(A)$ Stainless etl EFMA, dist:

481

482 Gold-Silver. EFMA [ig] d-Lupper. EFMA

485 Iran-Silican EFMA

$485(A) \quad 5 \%$ Austenite in Ferrite 486

487

$4 \%$ 15\% Austerite in Ferrite $30 \%$ fiustenite in Ferrite Iron Lartide in Ferrite

\begin{tabular}{|c|c|}
\hline 794 & Unel Trued Eu ([u J) \\
\hline $4 \% 5$ & Unal 1 ared Eu \\
\hline $4 \% 6$ & UnE1 1 Gred [U] \\
\hline 496 & Unal10red Eu ([u V) \\
\hline $79 \%$ & UnE17Gred Eu (EU VI) \\
\hline 00 & Unallared Lis (Lid VII) \\
\hline .07 & Fatassium Feldspar, TE \\
\hline 610 & Diass, Trace Elem 500 fFm \\
\hline 1.1 & Dilass, Trace Elem 500 fPm \\
\hline 2 & Gilass, Trace Elem So fFm \\
\hline & Trare Elem 50 pPm \\
\hline 61 & Gilass, Trace Elem 1 FPm \\
\hline 61.5 & Gilass, Trace Elem 1. PPM \\
\hline 616 & Dilass, Trage Elem . OL PPM \\
\hline 617 & Diess, Trage Elem .02 PFm \\
\hline 62 & Souda Lime Flat [ilass Comp \\
\hline 621 & Contairier Diass, Dump \\
\hline 62 & Giass, Leaching \\
\hline 620 & Gontainer Gilase, Leaching \\
\hline & Gilass, Electrical Resist \\
\hline
\end{tabular}

ea

$\$ 5292$

ea

4. $5 \mathrm{mim}$

$\$ 3602$

set (G)

set (b)

+ 1.69

\$ 232

क 22

3 mim $5 q$

52

1. Em dist:

$\$ 32$

2 cm dist:

342

1 Em dist:

क 32

$\Xi \pi m$ disl:

\$ 144

rod

$\$ \quad 92$

rod

rod

riad

rod

+ 82

审 12

क 82

\$ 82

rod

$\$ \quad 82$

5

$3 \mathrm{~mm}$

+ 69

108

1. $m i m$

\$105

$3 \pi m$

\$ 100

1. mim

$\$ 108$

$3 m m$

108

1. $\pi i m$

\$108

$3 \mathrm{~mm}$

$\$ 109$

1. $\pi m$

\$ 108

set (3)

\$1

set ( 3

* 90

$2.21: 9$

112

$2.21: 9$

$+112$

$200 \mathrm{C}$

$\$ 120$ 


\begin{tabular}{|c|c|c|c|c|}
\hline 625 & Zinc-base $A$ & こロリコ「・ & 克 & 94 \\
\hline 626 & Zirn-base E & รquลrอ & $\Phi$ & 97 \\
\hline 627 & ZinE-tiase E: & 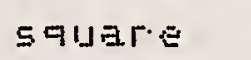 & $\$$ & $\Xi$ \\
\hline$\angle 20$ & Zinit-base & SqUar. & 中 & 97 \\
\hline 629 & Zinc-tiase $E$ & 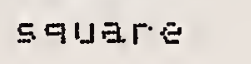 & $\$$ & 94 \\
\hline 630 & Zine-Lase F & 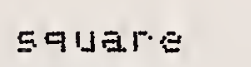 & \$ & \\
\hline 631 & Zine Speltar (mod) & square & $\$$ & \\
\hline 693 & Fartland Coment, rest & 1.59 & \$ & \\
\hline 694 & Fartland Eement, $901 \mathrm{~d}$ & 159 & $\$$ & \\
\hline 695 & Fartland Fement, blue & 1.59 & $\$$ & $=7$ \\
\hline 636 & Fartiand Eement, rel law & 159 & s & \\
\hline 67 & Fartland Gement, pint: & 1.5 두 & $\$$ & \\
\hline 600 & Fartiand Lement, arean & 159 & $\$$ & \\
\hline 69 & Fartland Lement, Elear. & 1.55 & 中 & \\
\hline $640(A)$ & 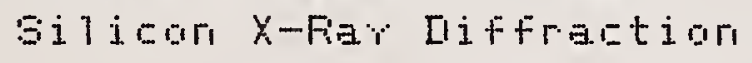 & 109 & 苦 & \\
\hline 641 & Ti-base A1 To\%, B川n (A) & Uisl: & 平 & \\
\hline 642 & Ti-tiase Allar, EMr (B) & dist: & $\$$ & \\
\hline 648 & Ti-kase A1 $10 \%, \quad B m n$ (E) & dist: & 中 & \\
\hline 644 & $T$ i-tase $20 \cdot-2 F=-2 M a$ (A) & di $\leq t:$ & \$ & \\
\hline 645 & Ti-bese 21 -r.-2Fe-2Ho (B) & 寸ist: & q & \\
\hline 646 & Ti-tase $2 L r-2 F-2 N_{0}$ (I) & di 5 : & 专 & \\
\hline$\Leftrightarrow .5(A)$ & $T i A 11 \Leftrightarrow, \quad 6 A 1-4 V$ & dj. 5 t: & $\Phi$ & \\
\hline 660 & $\operatorname{La} A$ Stee $1 \leq(661-6 / 5)$ & $r \cdot 0 i d s$ & 专 & 12 \\
\hline 671 & NiEl:el & 255 & \$ & 7 \\
\hline 672 & Nicke1 Dride 2 & 259 & tit & \\
\hline 673 & Wirtel mide & 259 & 串 & \\
\hline$G E O L 1(A)$ & Hi Furitr Flatirum & $10=\pi$ & 韦 & \\
\hline GEI.L1. & Moped Platinum & $105 \pi$ & 安 & \\
\hline SGILZ & Dofed Flatinum & $1 \pi$ & $\$$ & \\
\hline 60 & Zj.п口, High-Fur.itr & $e a$ & 本 & 1. 6 \\
\hline 693 & ZinEMetal & 르 & $\$$ & \\
\hline EFFi & $\nabla i g 1$ d, Hj.gt,-F'」r.j.tr & $r 01$ & $\$$ & $4:$ \\
\hline 6E5iw & Diold, Histı-Furitr & wire & $\$$ & 12 \\
\hline $\sin$ & Baselt Fabl: & 60 임 & $\$$ & 1.4 \\
\hline 699 & Fer.r.uEhromium $\mathrm{B}_{i}$ & 1009 & 卖 & \\
\hline 690 & J.ror Gree (Denada) & 1.50 5 & 中 & \\
\hline 1 & Feduged Iran Dride & 1009 & s & 14 \\
\hline 72 & Jror Gre (Labrador) & 1509 & 㭏 & \\
\hline 99 & Iron Gree (Nimtia) & 1509 & $\$$ & \\
\hline$\Leftrightarrow$ & Baukite (Surinam) & $\Leftrightarrow 09$ & 生 & \\
\hline
\end{tabular}


67

69

69

$700(1)$

$700(\mathrm{DI})$

$701\{[1\}$

702

709

705

706

709

709

710

711

712

719

71.4

715

71.6

717

71.9

720

$720(A)$

$724(A)$

726

729

$731 \mathrm{LI}$

$731 \mathrm{L2}$

791.LS

79

$736(A)$

737

$79 \% \mathrm{LI}$

$79 \mathrm{LL}$

$799 \mathrm{LS}$

740

741

742

748

745
Bauxite ([omini.can)

Bauxite (Alamaicari)

Alumina, Fedultion rirede

Faper, Light-sensitive

Fiper, Lisht-sensitive

Faper, Fided Strifs

Fiastic, Light-sens . 124.

Fiastie, Light-wens .060

Folrstrrene 1.791: mol wt

Foirstrene 25et mol wt

Dilass, Dotical Stress

Di]ass, DFtical Etress

Soda Lime Giass, Visgos

Lead biass, Viscosity

Lead lilass, frrreal Ft

Barium Diass, Anreai Fit Aluming. Giass, frrieal $\mathrm{Pt}$ Alumiria bilass, Arrieal Ft Neutral Giass, Arrreal Ft Hi Euron Giass, Viseosity

Alumina, Elasticitr

Eafflire, Het Dapacitr

Tr.is, Basimetric

Tris, Heat of Golution

Selenjun; inter. Furjto

zing, Intermediate Furity Eorasiliate biass, T EN Borosilicate Dilass, T Exp Borosilicata biass, T ENF Ag-Au Thermogufle wire

Eopfer, Thermet Exp

Turigsteri, Thermal ENF

Fused Eilica, Th ENP

Fused Gilica, Th E\%F

Fused Silica, Th ENP

Zine, Defining Fised Fit

Tin, rletining Fixed Fit

Alumina, Melting Foint

Merourr, Fresedng Forint

[Gold, Vafor Freseure
609

609

60

pl:s 100

PFE 100

ENE: $1 \mathrm{t}$

plis:

Fl:

5

199

set (2)

$500 \div$

$900 \mathrm{at}$

1.3 1:c

225

2259

2259

2009

$250 \div$

4509

Ea

155

50

509

450 의

$450=$

2 i.

4 in

6 i.

$3 \pi$

2 in

2 in

2 j.n

4 in

$\Leftrightarrow$ in

$350 \%$

350 :

109

680

$15 \mathrm{~cm}$
$+82$

\$ 62

$+82$

+ 81

\$5

\$ 196

$\$ \quad 81$

$\quad 81$

\$ 134

$+69$

\$1.17

111

$+101$

\$ 139

* 77

$\$ 77$

* 77

$\$ 77$

$+77$

$\$ 130$

* 29

101

* 119

क 81

+ 89

$+56$

1. 15

+ 132

क 49

+ 198

(5)

s 115

+122

\$ 195

\$ 26

15

+ 188

* 111

\& 1.98

\$ 156

* MATEFIAL IN PREFAFAT TIN 
746

748

$760-1$

$763-2$

$763-9$

$764-1$

$764-2$

$765-1$

$765-2$

$765-3$

$766-1$

760

772

779

781. $[11$

79152

$B 0 \Xi(A)$

[1503(A)

$804(A)$

$805(A)$

$807(A)$

$808(A)$

809 (E)

E17(A)

$020(A)$

821

827

837

r.18:77

640

0840

[1941

849

1.84\%

850

1050

55

856

85

859
Cadmium, Vapor Pressure

Silver, Vafor. Fressure

A1, Mag Suscept, Gririder.

Al, Mag Suscept, wire 0.5

Al, Mag Suscept, rod $6 \mathrm{~mm}$

Fit, Mag Suscept, Grinder.

Ft, Mag Euscept, wire 0.5

Fd, Mag Suscept, crimider.

Fid, Mag Suscept, wire 0.5

Fid, Mag Susceft, sforge

Mrifz, Mag Suscept, cube

Low Temp Fixed Fits 0. 26

Ni, Mag Moment, gahere

Gilass Liquidus Temp

Mo, Heat Capacitr

Ma. Heat Capacitr

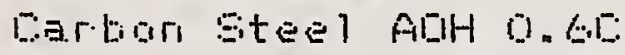

Carbugr Steel ACH 0.6E:

Carbon Steel Easja Elec

Lo A Steel, Mr 1.9

Lo A Steel, Er-V

Lo A Steel, Cr-Ni

Lo A Stewe1, Ni $\mathrm{S}$

Earban Steel, BLH 0.4L:

Jingat Iran

La A steel. Er-W

Lo A St1, Gr-Ma, SAE 4150

Tou 1 Stee 1, ErS-Moz-WS

Toal Steel, Ers-Moz-wa

Tad Steel, Er2-W1s-Da12

Tool steel, Er2-W13-[012

Tool Steel, WH-S AISI TI

S.S. Cr.5.5-pit.5

S.S., Cr.S.E-NiG.5

S.S., ERS-Ni2E

$S_{n} S_{n}, \operatorname{Cr} 3-N i 2 S$

Aluminum Dast fillor 556

Alumirum East Allor 000

Alumirium Allor bol1 mod

Aluminum Allor 7075
$6 \quad-m$

$5 \mathrm{~cm}$

$30 \mathrm{~mm}$

$250 \mathrm{~mm}$

$1.75 \mathrm{~mm}$

$30 \mathrm{~mm}$

E0 $\mathrm{mim}$

$30 \mathrm{~mm}$

$50 \mathrm{mim}$

19

$300 \mathrm{mim}$

set

2.4. $\mathrm{mm}$

7 FES

$10 \mathrm{Em}$

$10 \mathrm{Em}$

rod

dist:

rod

rod

r.ag

rod

rod

rod

rod

r.ad

rod

rod

dist:

rot

di. 5 :

dist:

rod

dist:

rod

dist:

30

509

359

359
\$114

\$ $12 \%$

+ 75

\% 69

; 1.53

\$11

क 32

\$ 159

496

+ 119

$\$ 115$

$\$ 2061$

क 160

$\$ 210$

$\$ 107$

$+161$

\$ 64

\$ 72

\$ 64

s. 64

$\$ 64$

$+64$

\$ 64

364

क 64

\$ 64

$\$ 4$

- 85

$+97$

* 85

4 97

क 97

* 85

37

\$ 85

$\$ 97$

+98

$\$ 98$

क 106

106 
871

972

874

875

979

E80 Nirlel Silver, ELIA 770

892

890

591

89

897

89

899

900

909

710

$911(A)$

$912(A)$

913

714

915

916

917

91.9

$91 \%$

920

921

92

923

924

925

926

927

928

929

$930($ II)

$931(\mathrm{C})$

934

935

96
Fhosphar Eronze, DLA 521

Fhosphor Ergrize, DEA 544

Eupra-Nicke1, DEA $706 \mathrm{HF}$

Dupro-NiElel, ELAA 706

NiEles 1 Silver, DELA 762

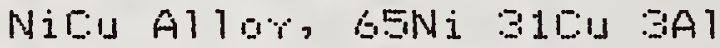

Ni-Hard, HL-25O+Y

Ni-Hard, TrFe I

Ni-Hard: TrPe IV

Trageal10\% A

Traceallar B

Traceal10r E

4 Aritiepilepsr Dridgs

Human Serum, El inical

Sodium Frruvate, [:1 irideal

Chalesteral, Dirical

Urea, Eliriseal

Ur.ic AEid, Elirical

Ereatirine, Elirical

Calcium Carburate, Dir.

Bilirutir, Dirnigal

Dilugose, E:1 inical

Fotessium Ehlaride, Elin.

Egdium Chlaride, Elirigal

Marritol, Elinical

Cortisol, Elirical

Tris, Elirigal-pH

Tris-HLI, DIiniEal-pH

Lithium Carboriate, Elir.

UMA, EIIRi[G]

Buvine Serum Albumin

Eovire Serum Albumin, 7\%

Lead Nitrate, Di irical

Magresium Diuconate, Din

Gilass Filters (Visible)

Liquid Filters LIV-Visitile

Clinical Thermometer.

Futassium Dichromate-LUV

Dujirire Gulfate Difrodrate
$100 \mathrm{~g}$

$100=$

$100=$

1009

$100=$

1009

1009

$150=$

1509

1.509

359

35

$35 \div$

set (4)

$\Leftrightarrow$ ViEls

25

29

259

$10=$

1.09

209

$100 \mathrm{mIg}$

259

309

309

505

19

259

359

$30=$

19

59

set (10)

$30=$

109

set (3)

set (12)

ez

159

1. 도
中

\$ E

+ 69

\$ 69

क 81

$\$ \quad 81$

क 84

\$ 101

$\$ 101$

क 1.01

\$ 163

क 163

163

+ 155

क 20

\$ 159

$\$ 73$

\$ 92

\$ 63

\$4

\$ 59

\$ 1.54

$\$ 2$

$\$ 77$

$\$ 66$

क 98

101

$\$ 77$

\$ 77

+ 89

\$ 91

$\$ 285$

每 175

\$ 67

\$ 97

+ 771.

$\$ 147$

\$ $54: 3$

\% 70

क 12 
597

739

745

946

947

949

$949(\mathrm{~F})$

950 (B)

951.

952

95

960

961

964

975

976

977

979

979

980

791-3 Lead Isatapic Fief

985

997

999

$9 \%$

991

99

95

90

999

$1001 \quad x-r a y$ Step Tatalet (0-4)

1002(c) Surface Flamimailitr

1.004.

1007 (A)

1008

1010(A) Mirrogopr Test Dharts

1017 (A) 01 ass Epheres 100-310um

$1018(A)$ biass Spheres 225-780um

1034

1035

Jron Metal, El juincal

Fiutarium Isotopir, $12 \%$

Plutarium Isatopilo, 19\%

Flutarium Isotapia, B\%

Plutarium Metal, Assar

Uranium Qxide, Assar

Boric Acid Assar-Isotopic

Boric Acid, Enriched E-10

Neutron Densitr Monitar.

Uranium Metal, Assar

Fiss Track Dilass L1-.07FFm

Chlorire Jsotopia Fief

Doffer Isotofie Fef

Bromine Isotofig Fef

Silver Isotapie fiet

Chromium Jsotofie Fef

Magresium Isatafie Fef

Fatassium Assar-Isotopic Strontium Assar-IsotofiE

Fherium Assar-Isotopic

Si.1 jogr Assar-Isotopic

Lead Assar-Isotopic

Uranium-205 Soln Spite

Uranium-203 $501 \pi$ Spile

F1utarium-244 Spike Assar

KL1-Frimarr Chemical

bilass Splieres 34-1.20um

Smote Derisit\%, Fiastic

Phota step Tablet (0-4)

Lriallored Copper.

Cofper-base Allor
509

159

5

0.259

0.259

0.259

0.59

259

$100 \mathrm{~g}$

0.259

wir.e

26 g

set $(b)$

set (b)

0.25

0.259

0.259

0.259

$0.25=$

0.259

set

19

1. 무

pl:g (50)

ea

ea

1.55

109

1. $\mathrm{mi}$

609

Ea

set (4)

$6 \Xi 9$

set (3)

ea.

set (5)

84 a

74 의

rod

509
$\$ 60$

* 95

\$ 477

$\$ 270$

+ 271

\$ 206

\$ 559

$\$ 187$

$\$ 106$

$\$ 72$

\$ 72

\$ 195

\$ 81

\$ 81

\$ 81

\$ 91

$\$ 81$

$\quad 81$

\$ $\theta 1$

$\$ 81$

$\$ 190$

क 33

事 74

122

+ 132

+ 175

\$ 209

* 187

$\$ 361$

\$ 92

\$ 195

$10 \%$

$\$ 85$

$\$ 70$

\$ 195

\$ 45

\$ 74

$\$ 74$

\$ 54

क 84 
1056

-1051 (E)

$1052(B)$

$105(\mathrm{~A})$

1055 ( $B$ )

$1057(\mathrm{~B})$

$1060(A)$

$1061(1)$

$1062(B)$

$106(E)$

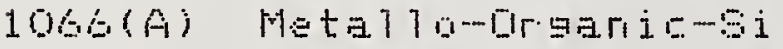

1069(E) Metal1

1070(A) MetalTa-Dragris-Sr.

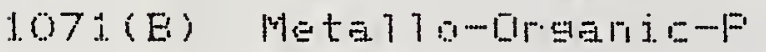

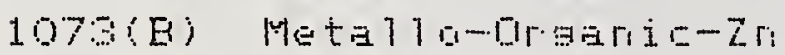

1074(A) Metal7g-Dranja-Ga.

$1075(A)$

$1077(\mathrm{~A})$

$1079(\mathrm{~B})$

$107 \%(\mathrm{E})$

1090 (A) Metal7 a-Drgari=-[1]

1094

1095

106

1007

1085

1069

1.090

1092

109

1094

i. 102

$110 \%$

1.1104

1. 106

$1.10 \%$

1107

1.1107

1108

1:110

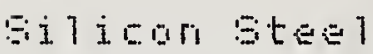

Meta 11 y-Organig-Ea

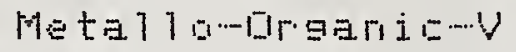

Meta 17 a- Dr.

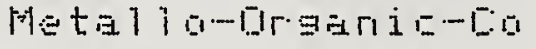

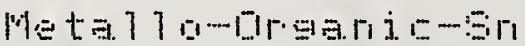

Meta $170-i r$ and

Meta i 1 a- Dregari $=-M$

Meta. $110-[1 \cdot a \operatorname{arj}=-M j$

Hetel 7 o-Drganio-Al

Metal 1 a-Drgani $E-A=$

Meta? 1 a-Orgariar

Metal 17 G - Mrearia-Fe

Wear letale ir Gil dorform

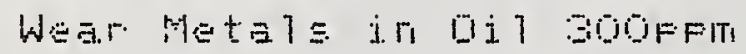

Wnal Tored Tij, Hrober

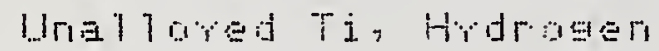

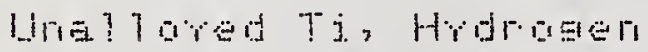

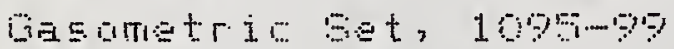

ligat Trori, lorem

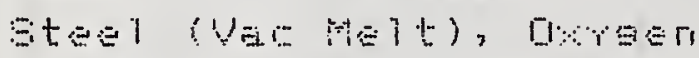

Gtes (valve), rrat

Stee (Maredrg), Dryen

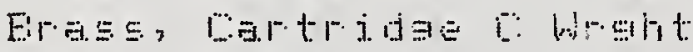

Sress, rres Cut A wrot

Brese, Fres rut E wh lest

Erass, Navai A brett

Buss, Mavel A Chl Bet

Brass, Navil E wrotht

Er Ese, Mavel B Dhl lost

Erass, Niaval : Wrott

Erass, Maval lo bri lot

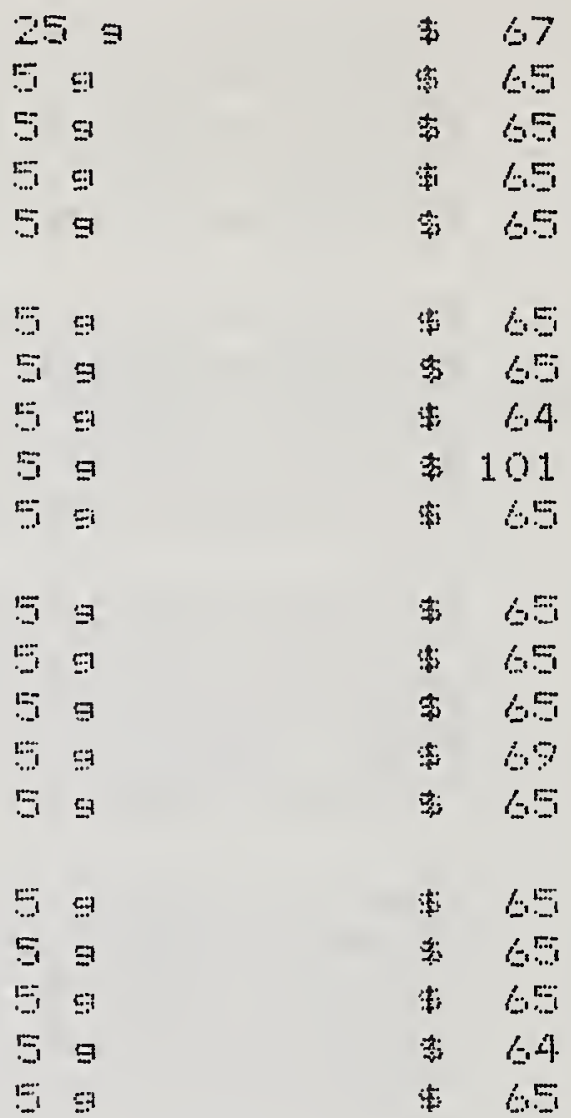

5

$85 \mathrm{mi}$

$95 \quad$ m?

$10 \quad 5$

109

$10 \leq 1$

rod $0(5)$

rig

rogid

rog

65

(4) 146

क 181

\& 61

a 61

(4)

卖 12

\& 106

$+10$

\$ $10 \%$

rot

dis

d $\dot{i} \equiv \mathrm{t}$

equar

di $\leq t:$

5106

अ 122

$3 \quad 12$

等 122

需 122

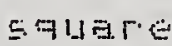

+ 122

di $\leq$ i:

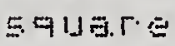

* 12

婉 12

건:

3 12

\$1 22 
1.1.19 Erass, Red A Hromt

C.1109

1. 1.10

E 1110

1.1.1. 1 .

-1.1.11

1. 1. 12

‥1112

1. 1.3

E1113

1.1. 1.4 .

$1: 1114$

1.1. 1.5

E.1.1. 5

1.1. 16

[.1.1. 11.

1.1. 1.7

ㅁ.11. 17

1.1. 1.

[‥11.15

1.1. 19

10119

1. 12

ㄷ1를

.1123

1131

1. $1 . \cdots$

11. 34

1. 1.25

115

$1130(A)$

$1130(\mathrm{~A})$

1. $1.4 .6(\mathrm{~A})$

1. $144(A)$

1. 1.45

. 14.6

1. 1.50

두 151

1.11 15

$\div 115 \%$
Br.

Br.esc, Fied $B$ wrotit

Erass, Fed B lihl Est

Eress, Red W.: Wroht

Brass, Fin E: EHT ISt

riildjug Metal f whet

Bilding Meta? A Lh! Est

Dii 1 djock hetal E Wraht

Diloing Metal E Lhl Est

Dilding Metal G: Wrot

\section{Gilding Metal E: Eh] Let}

Bronzes, Gml A Wratit

Eromze, [mil A [.h] C:st

Bronze, Bir B Wrat

Eroriza, Emi B Eth Est

Brones, Din G. Wretit:

Eronze, Emi C: Lil Est

Er ass, Al f Wretht

ErEss, A1 A CHI EEt

Bives, Al E Wrett

Brass, Al E Cthl Lst

EE LU, CAEFA 2E-72 Hrght

$B=-[1$, CABFA 2072 [. 1 :

EE-WU, EABFA $10-75$ bH

Sulder, Fbbo-5r40

Benring Metal, Fb-bese

Steat, Himizag-A10.9

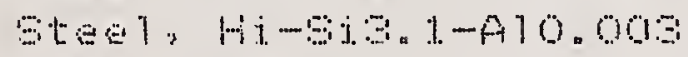

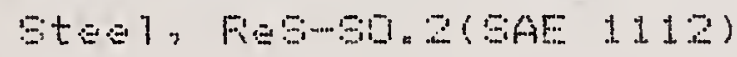

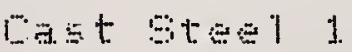

[ast steal

]. ror Flast Furraged

Tron, Fitast Furrates 2

White 1...e.t Trom

White cast Irom

whits arat dror

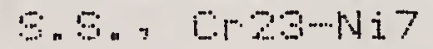

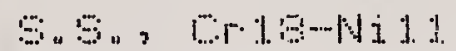

G. 5, ard $7-N .9$ 日j. $5 .:$

squars

dj. 5 :

S9uare

a $i \leq k$

122

क 122

4 122

s 122

$\$ 122$

5quare

* 12

di 5 :

5 9 u.

dj. $\leqslant$ :

5quare

+ 122

$\rightarrow 12$

क 122

+ 12

dj. 5 :

squares

disl:

Squar

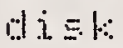

422

क 122

皮 12

* 122

क 122

드미부을

dist:

square

dist:

도일

* 122

क 122

去 12

乘 12

家 12

di. 5 :

squere

i. $i \leq 1:$

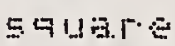

54uere

* 122

412

$+122$

* 12

$+122$

dist:

dis:

$d i \leq k$

ij.

dist:

dj $51:$

disl:

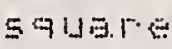

square

id 다:

$d \mathrm{j} \equiv \mathrm{l}$

dj. 5 :

di

di $\leq k:$

dist:

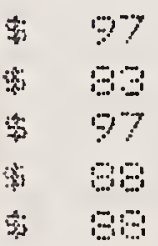

综 109

$10 \%$

察 12

12

+ 9

* 9

4:

* 11:

(5) 11.1

郝 $11 \%$ 
$1: 1154$

$-1155$

1156

1157

1159

$115 \%$

1160

1.166

$1169(\mathrm{~B})$

1. $1.70(\mathrm{~B})$

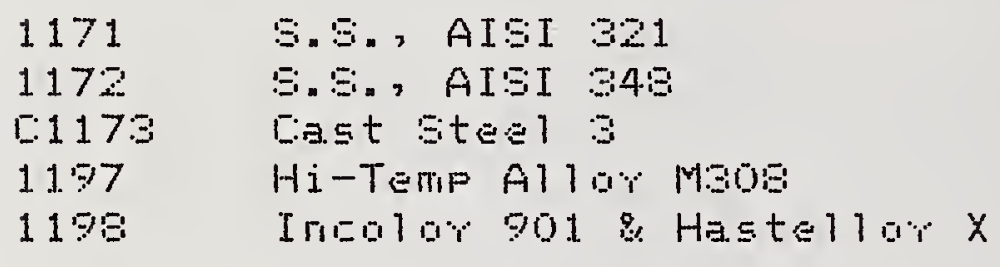

$11.9 \%$

$1207-1$

$1207-2$

$1205-2$

1.1. 21

122

1224

1226

1. 234

1235

1. $2: 0$

1237

1. 23

123

ㄷ. 1251

$1: 125$

ㄷ.125:

1254

1. 25

1256

1259

1259

1.2(1. (A)

$1262(A)$

$1263(A)$

S.S., Er. $19-N i 13$

S.S., AISI 316

Stea1, Maragira Nils

Toul SteEl, AISI. MZ

Hi-A Stesl, NiSG

Ele日/Mag Al10, Ni49-FeS1

Eler/Mag, Niso-Mo4-Fe14

Jrigat Irom $F$

Stee 1, Leaded 0.3 Ft Drir

$\Xi .5 .$, SAE $: 03$ SE

Hi-Temp A119\% LGo5 a ES16

Hi-Temp, Waspalor \#1

Hi-Temp, Waspala

Hi-Temp, InEG 719 \#2

Eteel, AISI 1211(mad)

La A Steel, AISI 9640

Stest, AISI 1079

Eteel. HY 130 Al1

Zir.onium A

Zirconium $\mathrm{B}$

Zir.gnium E:

Zir.Eal

Zir.ą $\because \because-4 \mathrm{E}$

Zir.ż日-4 F

FHos-LU (EII VIII)

FHos-[:] $([1] \quad I X)$

Pros-ing (10.1) $x$ )

LEI Eium in SteEl

Alumirum Gat All a\% 356

Alumirum Cest Allor 380

Aluminum Allar 6011 .

Aluminum Al $10 \% .7075$

La A Stee1, AISI. 4340

Lo A Steel, AISI 94817

La A steel, $[F-V(m a d)$ 寸ist:

dj.st:

dist:

dist:

dist:

Uist:

寸isk:

才i 5 :

dist:

dist:

寸ist:

寸isk:

dist:

dist:

寸ist: (I)

\$ 111

* 105

\$ 122

क 31

+

\$1.2

- 12

+ 122

\$ 5

4 65

果

中 $\quad 3:$

क 106

\$ $\quad 57$

+ 174

dist $(2)$

\$ 1.74

square

square

square

dj. 5 :

\$ $10 \%$

$+109$

+ $10 \%$

+ $7 \%$

disk:

寸ist:

日i $\leq$ :

di $\leq 1:$

disk

古 77

$\$ 50$

$\$ 70$

4 415

+ 415

dist:

di $\leq$ :

dist:

iㅗ:

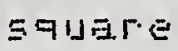

4 415

+ 415

क 405

* 415

4 130

square

क $1: 0$

+ 193

* 50

+1.3

+ 133

dist:

+119

disk:

dist:

di $\leq$ :

dis 5 :
+ $11 \%$

क 102

* 102

+ 100 
12E4(A) Hi Garban Etes (mod)

$1265(A)$ Electralrtis Iran

1267

1269

E. E., AISI. 446

1270

Lo A Steel, AISI 1526

Etee1, Gro-M1. (A:BG)

dist:

\$102

寸ist:

102

日ist:

事

dist:

t 120

dist:

क 119

1275

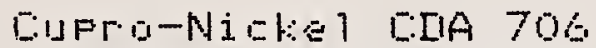

1. 276

Eupro-Nigkel EDIA 715

ㄷ.1255

Stesl, A242 A11 a\%

126

EteEl, HY BO Al $10 \%$

ㄷ12:7

HiA Stl, Al:I HK (:10 mad)

dist:

$\$ 91$

dist:

dist:

dist:

4 91

$+106$

\$ 90

寸is:

$+10 \%$

1.1. 219

HIA St1, AL:I EN-7M A-74:

[1:129?

HiA Et 1, AE:I E:A-SNM

$1: 101$ ( $\mathrm{A})$

Eur Lost/Bt 1

$2.511 \pi m$

$1: 202(A)$

Cul:r Loat/St]

$6 \quad 4 \pi$

$1: 30(A)$

EuEr Loat/st]

$12 \quad 15 \pi$

$1904(A)$

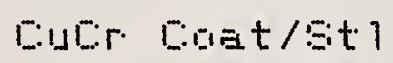

$2015 \pi$

$130 !(A)$

Lubropt/Et

$2515 \pi$

$1: 06(A)$

[uER [oat/St

$4011 \pi$

$1: 07(A)$

[uEr Eogt/St]

5101

$1: 0 B(A)$

[uL:r Diat/St

$6515 \pi$

1.:1O(A)

[is]r [ogt/St 1

50 $13 \pi$

$1: 11(\mathrm{~A})$

[uEr Eoat/st]

140

EuLr Loat/St

140 1 15

$1.312(A)$

Eis:r Elot/Bt 1

200 गान

$1313(A)$

EuEr Eoat/Et?

$250 \quad 11 \pi$

$1314(A)$

$400 \quad 11 \pi i$

$101(A)$

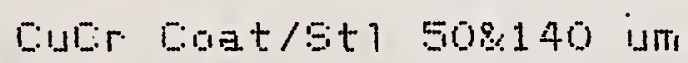

1. $:=2(A)$

Ni. [ont/Str 920 im

$1053(\mathrm{~A})$

Ni Cont/St 25060 um

$1061(A)$

Euler Enat $6,12,25,50$ um

$19(2)$

Eulir $40,80,140,800$ um

$130(A)$

E.JE: 200,400,

Hist:

年 109

dist:

set (4)

$+10 \%$

set $(4)$

set (4)

生 150

* 150

(⿻) 150

cet (4)

$\$ 150$

set (4)

set (4)

$\$ 150$

\$ 150

set (4)

完 150

set (4)

150

set (4)

set (4)

set (4)

set (4)

set (4)

15

+ 150

\$1.51.

$+154$

$+150$

$1364(A)$

EuCr $920,1000,1500,2000 u m$

$1365(A)$

Ni Coat/St $3,5,15,820 \mathrm{um}$

set

150

set (4)

\$i 150

set (4)

set (4)

set (4)

$\$ 150$

150

$+150$

$1060(A)$

Ni Cont/St $25,35,40,50 u m$

$1.667(A)$

Ni an Brass $3,9,16,825$ uाm

$1370(A)$ DuEr Eoat/St 200ta1500um

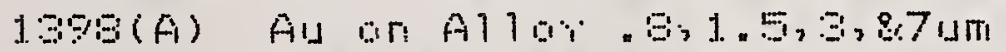

set (4)

部 1.50

set (4)

\$5

set (4)

क 151

set (4)

$15: 5$

set (4)

\$1 $5:$

$1450(\mathrm{~B})$

1.460

Thermal Fesistange Fitier.

set (E)

$20 \%$

set (4)

4

ea

$\$ 410$

S. 3. Th Thid-Fiesist

1461

S. S., Th Eard-Fiesist

$\because \quad$ i.

\$ 1.4.

* 169 


\begin{tabular}{|c|c|c|c|c|}
\hline 1462 & $\Xi_{n} \Xi$, Th EDrad Fies & 2 in & $t$ & 213 \\
\hline 146 & ].rin, TH Gad-Feset & $2 \quad$ i. n & s & 1. 36 \\
\hline 146.4 & Trori, Th EGTd-Fesist & $2 i r_{1}$ & 2: & $19 \%$ \\
\hline 1. $4.6 \%$ & Turastari, Th Lund-nesset & $\therefore \quad$ i. 11 & 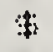 & 1.48 \\
\hline 1466 & Turasteri, Th Eond-Fesist & 2 in & 蓉 & $17:$ \\
\hline 1. 467 & Turiseteri, Thiorid-Fiesjet & $\therefore$ i. $1 r_{i}$ & 耓 & $10 \%$ \\
\hline 1465 & Turasteri, Th, Lond-Feseist & $2 i \Gamma_{1}$ & t & 176 \\
\hline 1. 469 & Turiseten, Th Fond wesist & $\therefore$ j. n & 管 & 209 \\
\hline $147 \%$ & Dias Ferrme日tar Fal\% Fi $1 m_{1}$ & $50 t \quad(15)$ & $*$ & 170 \\
\hline .1. 475 & Falvethitene, 52l 1701 wt & 5 & s & 101 \\
\hline 1476 & 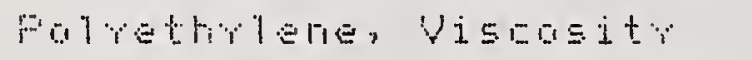 & 505 & \$ & $13 \%$ \\
\hline 1.47. & 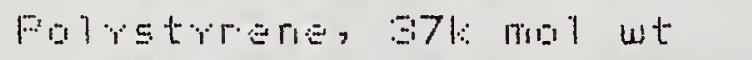 & 2 & s & 196 \\
\hline 1479 & Fulystrene, Iri mol wt & $\ddot{2}$ & s & 125 \\
\hline 48 & Privethrlene, 14t: mil ut & 1. $\mathrm{E}$ & $\$$ & 1.79 \\
\hline 145 & Folrethrlene, $22 k$ mol wt & $i \leq$ & 掌 & $17 \%$ \\
\hline 434 & Falretrilene, 120l mil wt & 1.9 & 4 & 17 \\
\hline $4 \% 0$ & Frijsotutrlene, Fhen & $250 \mathrm{~mL}$ & 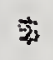 & 25 \\
\hline 49 & Lu Vjegostr Fubber & $34 \quad 1: 5$ & $\$$ & 20 \\
\hline 1511 & 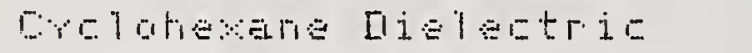 & $400 \pi \mathrm{L}$ & 客 & 22 \\
\hline$=12$ & Dish or oetherae rijelectria & $400 \mathrm{HTL}$ & \$ & 215 \\
\hline 1515 & 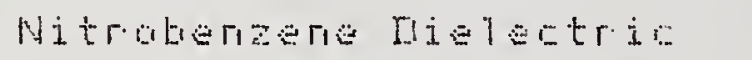 & $400 \mathrm{~mL}$ & 档 & 215 \\
\hline 1516 & FEF Fon & E. & t & 337 \\
\hline 1517 & 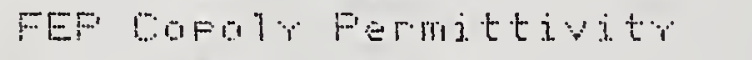 & $E=$ & 荬 & 337 \\
\hline 19 & 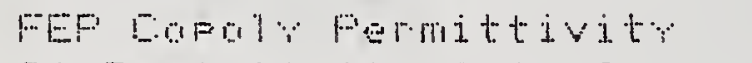 & $\Xi \Xi$ & sis & 307 \\
\hline 31 & Gi Fasjetivity, $1-10$ & $\equiv \pm t \quad(2)$ & 案 & 414 \\
\hline 22 & Gj Fesictivitr $20-7-190$ & Eet $\quad(\because)$ & 蜜 & 59 \\
\hline $1=-2=$ & Si pesistivitro.01-1 & $\Xi \Xi t \quad(2)$ & 等 & 414 \\
\hline 15.2 .1 & Tron mostrauer & 트. & 察 & 24.1 \\
\hline 156 & Drster. Tissue & $30=$ & $\$$ & 94 \\
\hline 1607 & Whent Find & 80 & 慗 & $10 \%$ \\
\hline 1.56 & Fise Flour & $\equiv 09$ & t; & $10 \%$ \\
\hline 10 & Drewers Yeset & 50 & $\$$ & 91 \\
\hline 1571 & Dicherd Legves & $10=$ & 寞 & 5 \\
\hline 192 & ロitrus Leaves & 70 & 邦 & 1.06 \\
\hline 1573 & Tomato Lenges & $70=$ & क & 107 \\
\hline 1. 5.75 & Pine Nesdes & $70 \leqq$ & 㲅 & 1.17 \\
\hline & Brving Liver. & $50=$ & + & $12 \%$ \\
\hline & 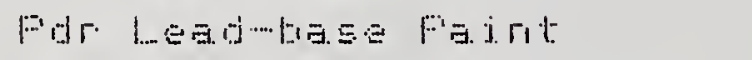 & $3=1$ & 策 & 心. \\
\hline 50 & Stinta Dil & set $(5)$ & $\$$ & 204 \\
\hline & PEE & $\leq t$ & 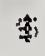 & J. \\
\hline
\end{tabular}


1.590

1590

1.600

1619

$1620(A)$

$1621(B)$ Sulfur in Fuel Dil $1 \%$

1(22(B) But fur ir Fuel Dil $2 \%$

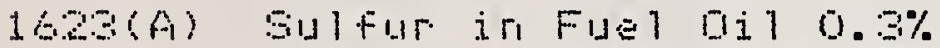

$1624(A)$ Sulfur ju Dist Dil $0.2 \%$

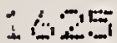

160

1627

$162 \%(A)$

1630

1. $62(A)$

1. $19(\mathrm{~A})$

$164(A)$

1635

$1.36(A)$

$1637(\mathrm{~A})$

$169(A)$

$164 \mathrm{j}(\mathrm{A})$

16. 2 (B)

$1649(A)$

1644

1.645

1646

$164 \%$

1649

1649

$165:$

165

160

1654

165

$16=9(A)$

$1609(A)$

16 OC(A)

$16 \in \mid(A)$

$A \Leftrightarrow 2(A)$

Stabilazed wine

2 Ariticunvulsarit [r.ugs

Magretic Tape-Lassette

Buifur in Fuel Dil $0.7 \%$

Eulfur j. FuE

Sol Fermeatiar, Tute

602 Fermation Tute

S12 Fermeation Tube

1402 Permeatjar Deviae

Mereur in Eoral

Trane Elemente in Gol

TE ir GOE! Fl\% ASh

Trence Elemerts/Fuel Gi.l

Trage Eisments in Goal

Legid in Feference Fuel

Lead in Fieference Fual

Lend in Fiefererice Fuel

Merour.r in Water-Goricentr.

Mer eur in Water-Trace

Trace Elements iri Water.

PAH Generator alums

Fiver Sedimert

Estuaririe vediment

Friaritr Fal utant FAH S

Urban Farticulate Matter

Urban Must/Grganics

zrea Ghromete Heat Goures

ZrBa Chromate Heat Source

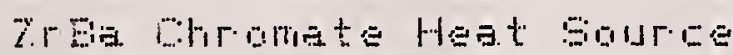

ouarta Heat of Solutiar

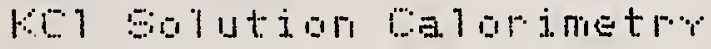

Methane ir Air,

1 FPM

piethane j.ri Air.

1.0 PPIT

Methare 是 Frofarie 431 FFm

Eiliz j. 14.

500 PPM

$6 \mathrm{~Hz}^{2}$ i. $\mathrm{NI}$ ?
1000 PPT set $(10)$

set (4)

ex

$100 \mathrm{~mL}$

$100 \mathrm{~mL}$

$100 \mathrm{~mL}$

$100 \mathrm{~mL}$

$100 \mathrm{~mL}$

$1.00 \mathrm{~mL}$

$10 \mathrm{~cm}$

$\because \quad=m$

$25 \pi$

$10=m$

509

$75 \div$

759

$100 \mathrm{~mL}$

759

set (12)

set (12)

set (1:)

set (a)

$950 \mathrm{niL}$

$950 \mathrm{~mL}$

set (S)

70 므

759

$\therefore \quad m L$

2

109

$50 \quad 9$

509

50 도

259

$30 \leqq$

E 1

$=1$

E 1

Er

Er 1
\$ 121

क 155

4 259

$+10 \%$

$\$ 109$

$\$ 10 \%$

\$ $10 \%$

+ $10 \%$

\$ $10 \%$

\$ 195

\$ 201

\$ 191

$\$ 279$

\$ 67

+ 1.14

$\$ 131$

- 1.66

$+114$

4 16

* 116

+1 116

\$ 117

\$ 1.49

事 1 目

\$10

* 144

+1.22

$\$ 127$

\$ 134

\$ 175

\$ 84

事

\$ 84

+ 279

क 152

550

$\$ 500$

क 590

+ 590

\$50 


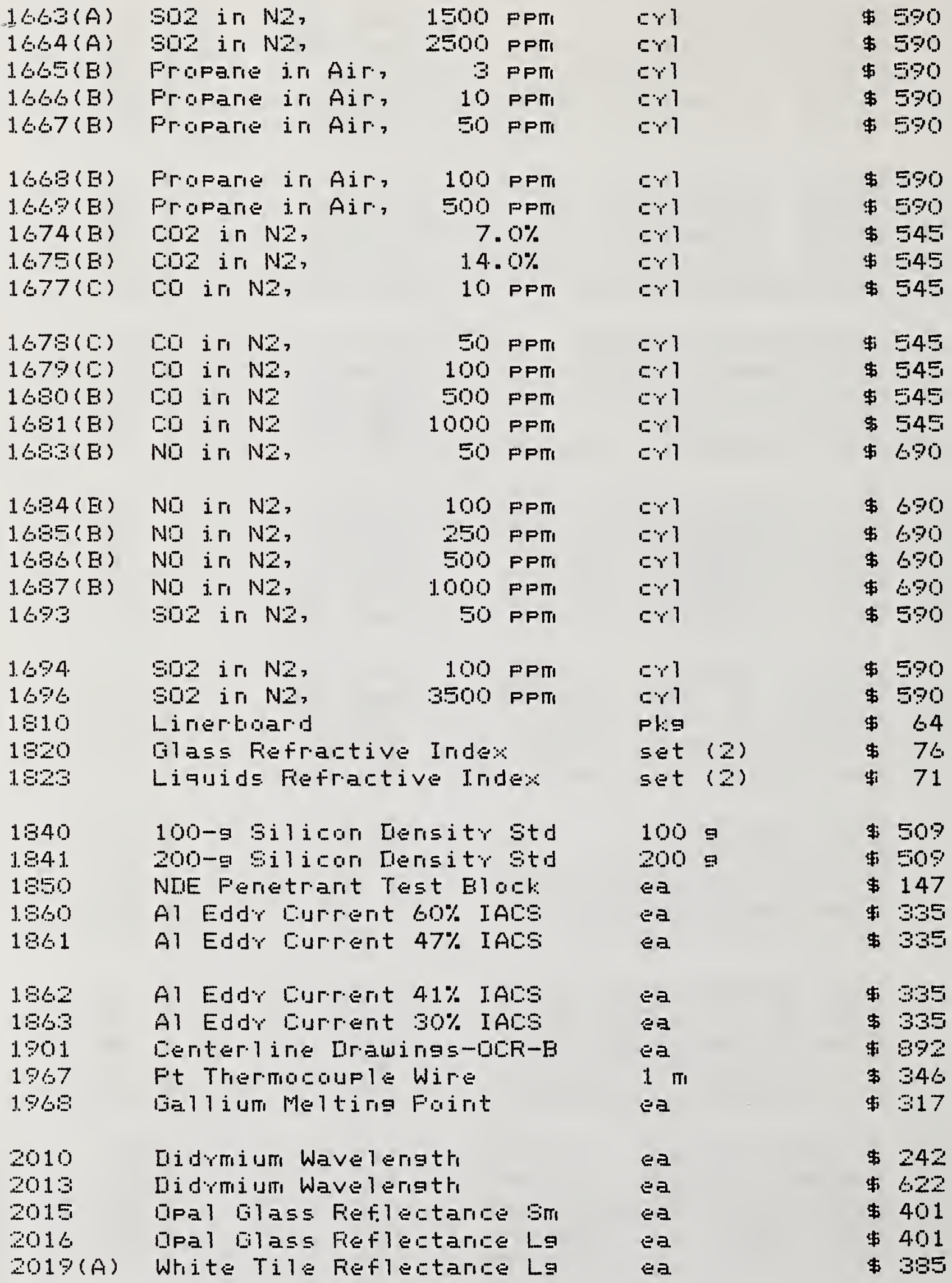


2020

2021

2022

2023

2024

2025

2030

2031

2032

2061

2106

2107

2141

2142

2143

2144.

$2186 I$

21.86 I I

2191

2192

2201

2202

2203

$2303(A)$

$2319(A)$

$2338(A)$

$239(A)$

2601

$2612(A)$

$2613(A)$

$2014(A)$

$2619(A)$

$2620(A)$

$2621(A)$

$2622(A)$

$2623(A)$

$2624(A)$

2625

$2626(A)$

2627
White Tile Reflectarice Sm Blk Eriamel Fieflectarice Lo B1: Eriamel Fieflectarice Sm Al Secorid Surface Mirror. Al Second Surface Mirror.

Al Mirror with Wedge Giass Filter (30\%T)

Metal on Buartz Filters KI Strar Light

Fieflectarice Step Tablet

Centroid Color Charts

Eentroid Dolor Kit

Brea, Microchemical

Bromoterizaic Aatd Micro

Fluoroberzaic Acid Micro

Chooraberzaic Acid Micra

Fot Iihrdro Fhosphate $F$

[isod Hrodro Fhosphate p[l

Godium Bicartoriate, FII

Sodium Carbariate, $P[1$

Sodium Chloride, pNa plE1

Fotassium Ehlaride pk plol

Fotassium Fluoride, PF

Au/Lamiriate . $8,1.5,3,7 u m$

Au/Lu 0.8,1.5,3, $27 \mathrm{um}$

Sn/St1 $2.9 \& 1.6 .51 \mathrm{um}$

Snlst 1 1.5,4.1,7,8,19um

Rutur EFF Atsorption

CO in Air.,

Do in Air,

Do in Air.

EOL in N2:

$\mathrm{CO} 2$ in $\mathrm{N2}$,

Colz in $N 2$,

002 in $\mathrm{N2}$,

002 j. $N 2$,

002 in $\mathrm{Nz}$,

[0.02 in 12 ,

[012 in N2,

NII in
9. EFFII

1.5 PPII

$4: 3 \quad$ PPII

$0.5 \%$

ea

ea

ea

ea

ea

ea

ea

set ( $:$ )

259

ea.

tit: $1 \mathrm{t}$

set

29

29

29

29

309

309

309

309

1259

$160=$

1259

set (4)

set (4)

set (4)

set (4)

set (2)

Er

Er1

$1.0 \%$

$1.5 \%$

$2.0 \%$

$2.5 \%$

$3.0 \%$

$3.5 \%$

$4.0 \%$

5 PPII
385

क 385

क 365

क 509

\$ 509

$\$ 695$

क 314

$\$ 1260$

क 221

\$ 1.51

\$ 31

\$ 36

क 67

\$ 67

$\$ 7 t$

$\$ 67$

\$ 82

क 82

क 82

\$ 73

क 69

क 69

क 116

\$ 376

क 369

\$ 376

\$ 370

\$ 228

क 545

$\$ 545$

क 54.5

\$ 54.5

\$ 545

\$ 545

\$ 545

\$ 545

\$ 545

\$ 54.5

$\$ 545$

4600 


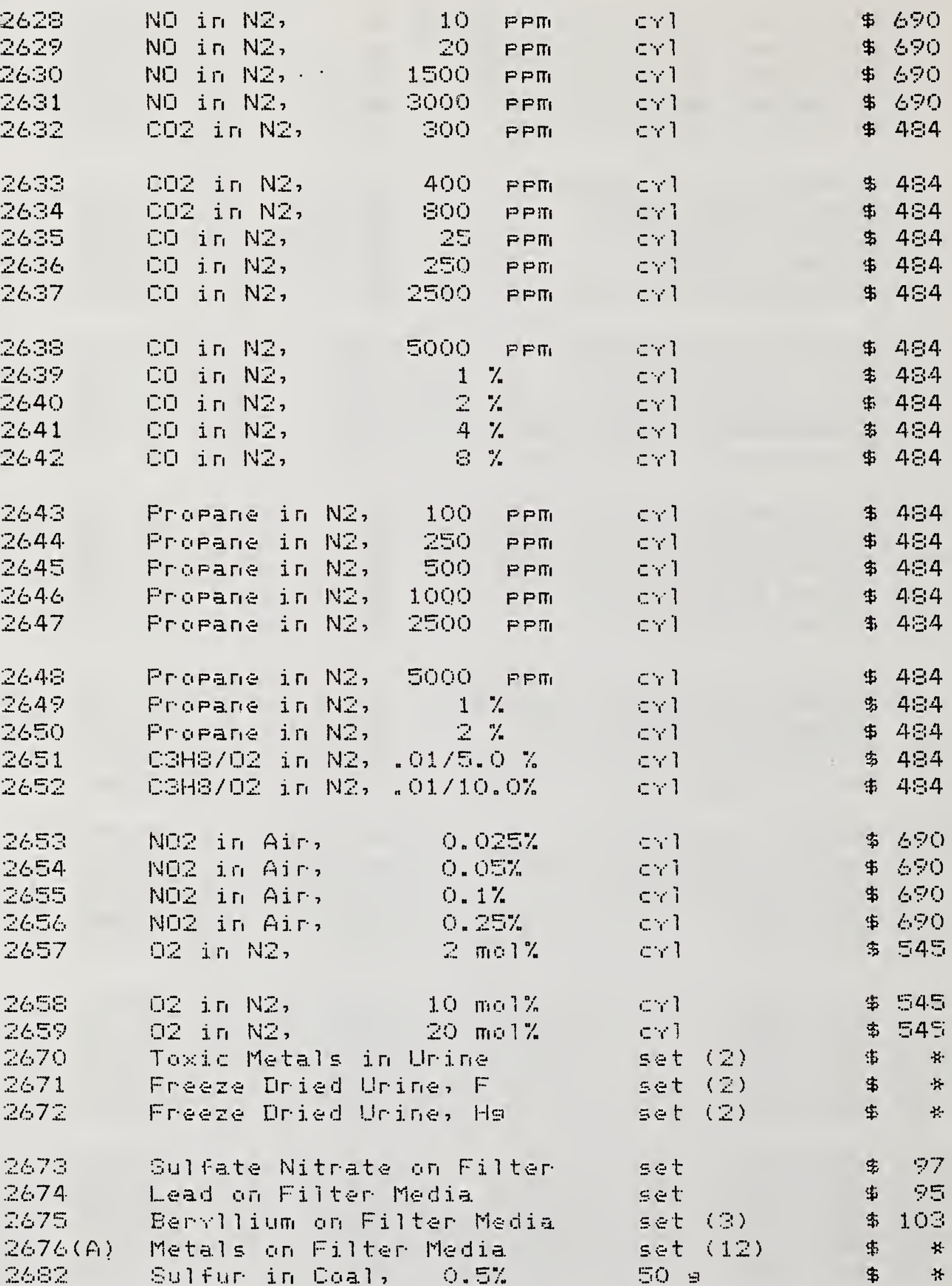

* MATEFIAL. TN FFEFAFATTIN 


\begin{tabular}{|c|c|c|c|c|}
\hline 263 & Sulfur din Eoal, & $50 \div$ & 4 & $k$ \\
\hline 2694 & Sulfur in Enal, & 509 & $\$$ & 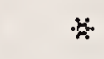 \\
\hline 2695 & 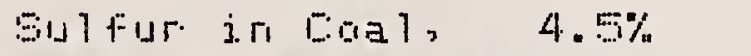 & $50 \leqq$ & \$ & $*$ \\
\hline 2200 & Magmetio Tapa-Fiesl & $E \overline{1}$ & & 164 \\
\hline 3210 & Magnetic Flenjble [ist: & e⿴. & $\$$ & 504 \\
\hline 3216 & Magrietio Tape-Lartridge & Ea & \% & 439 \\
\hline $4200(\mathrm{~B})$ & Les j. $14 \pi-1.7$ & $E \Xi$ & $\$$ & 1. 1.4 \\
\hline $4201(\mathrm{E})$ & $N$ i. & ea & $\$$ & 265 \\
\hline $4202(1:)$ & 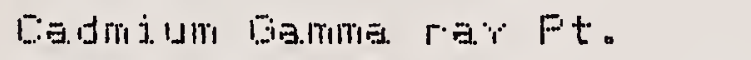 & $E \bar{E}$ & $\$$ & kk \\
\hline $4203(1)$ & $50 \cos 1 t-60$ & Ea & $\$$ & $* *$ \\
\hline 4204 & FHromium- Ft. Egusres & $e$ e & 生 & が \\
\hline 4205 & Thar ism-220 Giamma rar Ft. & 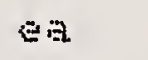 & 范 & 179 \\
\hline $4206(1:)$ & Thor i um/Tha 11 i uा -220 & ez. & 荬 & 29 \\
\hline 4207 & Lesidm-157-Bar i $4 \pi-157 m$ & $E$ & $\$$ & 11.4 \\
\hline $420 \%(1 ;)$ & Yttrjum-BE Faint GuurE & Ea & 中 & 275 \\
\hline 4210 & Eog $101+-60$ & $\mathrm{e} \theta$ & 客 & $* *$ \\
\hline 421.1 & Am:r. $=$ i. $1 m-241$ & Eᄑ & 幽 & 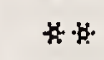 \\
\hline 4212 & NrFton & Ea & $\$$ & $24 \%$ \\
\hline 4215 & Amer.iEium-241 & E & $\$$ & 27 \\
\hline 4214 & 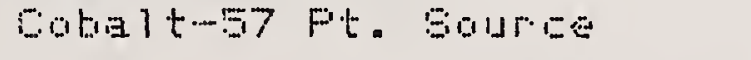 & $ت \exists$ & 密 & Ł。 \\
\hline $4215(F)$ & 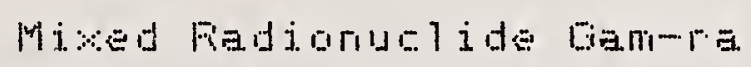 & 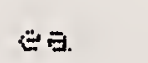 & $\$$ & 点必 \\
\hline $4216(E)$ & Mined FadianuElide Biam-ra & $E$ & 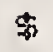 & 194 \\
\hline 421.7 & Ei1vㅍ-110m Pt. EvurEe & E酐 & $\$$ & $* 4$ \\
\hline $4218(1)$ & Eurofium-152 & E日 & 索 & 200 \\
\hline $42.9(F)$ & Cudaidm-10950istion & 5 & 忠 & $\nLeftarrow *$ \\
\hline 422 & Hexaderane-Cartion & $\Xi 9$ & : & $4 x$ \\
\hline 40 & 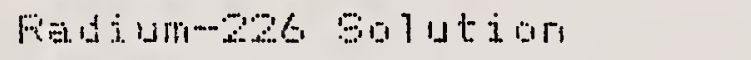 & 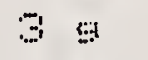 & $\$$ & $* *$ \\
\hline $42-4$ & Fajium 206 Solution & $\because 9$ & \$ & $* * k$ \\
\hline 4225 & Tin-113-1. ndsm-1. & 5 & $\$$ & \# \\
\hline 426 & Ni. $=$ t: $1-63$ & $4.1 \Xi$ & 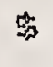 & 263 \\
\hline $429(\mathrm{~B})$ & 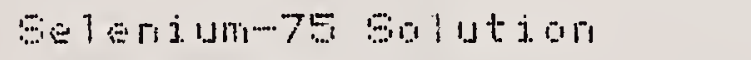 & 59 & $\$$ & *. \\
\hline 422 & A ? uri j. rustr-26 & 4.69 & 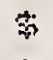 & 300 \\
\hline 4.230 & 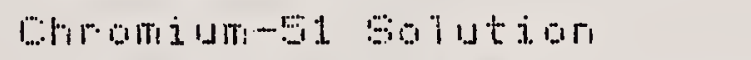 & & t & fH \\
\hline 420 & Giver-110m Sulutian & 59 & 䒠 & $x$ \\
\hline 42936 & 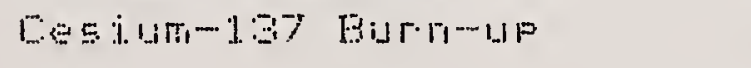 & $\because$ & 衿 & 437 \\
\hline 4234 & Strortium-Yttriumono & 3.19 & $\$$ & to \\
\hline 4230 & 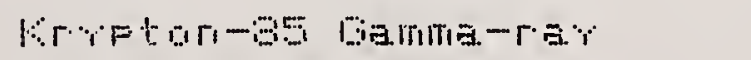 & 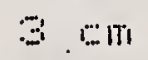 & 本 & 1.81 \\
\hline 4237 & 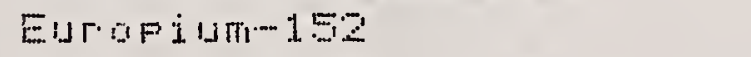 & ea & 卖 & k * \\
\hline 4240 & 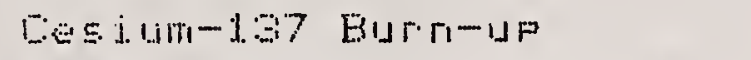 & $E \theta$. & $\$$ & $* *$ \\
\hline $424 .(5)$ & Bar-ium-1SO Foint Source & $E B$ & w & 30 \\
\hline
\end{tabular}

* MATEFITAL IN PFEFAFATTIOW

* FRICE AND AVAILAEILITY DN FEGIEST: (301)921-266 


\begin{tabular}{|c|c|c|c|c|}
\hline 4245 & Ear.tiar-14 Sialution & 59 & \$ & 1.50 \\
\hline 4246 & 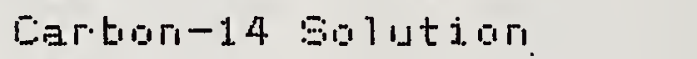 & 5 & $\$$ & 1.50 \\
\hline $4: 47$ & E:ar·bor-14 Salutian & 59 & $\$$ & $k$ \\
\hline 4249 & Bar.jum-Lantharıum-14i & 59 & 斗 & $\hbar$ \\
\hline $4250(E)$ & Eesium-134 Sulutinn & 59 & 卖 & 30 \\
\hline 4251 (E) & Barium-1SZSolutiar & 59 & $\$$ & 360 \\
\hline $4254(E)$ & Mixed Fiadiaruglide & $5 \mathrm{~mL}$ & t; & 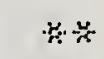 \\
\hline 4257 & Manganese-54 Salution & 59 & t & $\star k$ \\
\hline $4260(B)$ & Iron-ss Fhatan Emissiar & ea & 虾 & 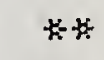 \\
\hline $42 \leqslant 1$ & 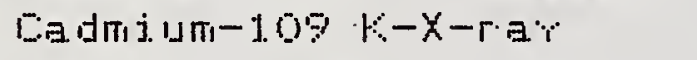 & Ea. & 斗 & 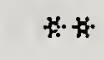 \\
\hline 4263 & Strontium-es & $E$ & q & $* *$ \\
\hline $42 \leqslant 4$ & Tin-121m Pt. Fiamma rar & EE. & 卖 & $\star *$ \\
\hline $4265(E)$ & Iadine-125 Frotan & $E \equiv$ & $\$$ & $* x$ \\
\hline 4266 & Variadium-49 Ft. Sour & Ea & \$ & 41.4 \\
\hline 4275 & Mixed FiadinnuElide & ea & 事 & 379 \\
\hline 4276 & Mixed Fationual ids & $5 \mathrm{~mL}$ & s & k゙ \\
\hline $4300(E)$ & $A r \cdot 90 r_{1}-37$ & ea & $\$$ & 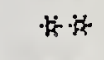 \\
\hline $4: 301(B)$ & Ar.9 $=r_{1}-37$ & ea & $\$$ & $\# *$ \\
\hline 4902 & $A r \cdot 90 r_{1}-39$ & $10=m \Xi$ & 䇺 & $19 \%$ \\
\hline $4: 304$ & $x \in n_{1} \pi_{1}-1.91 \pi_{1}, \quad 9 a 5$ & ea & 4 & $* *$ \\
\hline $4306(B)$ & Xerori-13:, gas & Ea & 荣 & $x+x$ \\
\hline $4307(1 \mathrm{i})$ & xen101-1:3, gas & $22.5 \quad 0 m=$ & 华 & tæ: \\
\hline $4305(B)$ & Hrotur, 55 , gas & 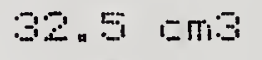 & ; & 102 \\
\hline $4: 309(E)$ & $x=n a n-127$, 포.5 & $325 \quad 5 m 3$ & $\$$ & 34 \\
\hline 4910 & Mixed Giasenus Fadionet & en & $\$$ & $\nLeftarrow$ \\
\hline $4: 30$ & Fu-23 Alpha-Partisle & 29 & s & $\star *$ \\
\hline $4: 31$ & Fin-23\% Alpha-Fiarticle & 29 & 专 & 1.51 \\
\hline $4: 32$ & Amer.i口ium-24S巨口! & 29 & 政 & k. \\
\hline 4330 & Amer.i口ium-24:5口1r & 59 & 宲 & $*$ \\
\hline $4: 5: 4(B)$ & Flutanium-2428n1 & $4 \mathrm{~mL}$ & 字 & 1. 40 \\
\hline $4: 35$ & Fu-242 Alfhie Farticle & $5 \mathrm{~mL}$ & t & $3 x$ \\
\hline 4935 & Plutorjum-240 Evlutian & $E$ & \$ & 467 \\
\hline $4350(E)$ & Fiver :Eediment, Enviran & $85 \equiv$ & 糔 & 15,4 \\
\hline 4352 & Humear Liver. & $45: 5$ & $\$$ & $=150$ \\
\hline $4: 5: 3$ & Fäbr Flats Soil \#1 & 859 & 卖 & 154 \\
\hline 4955 & Feruvian Ëil & $75 \subseteq$ & 要 & 1.50 \\
\hline $4: 51$ & Hudrogen-S Solution & $490 \quad \exists$ & 索 & 211 \\
\hline $4: 70(E)$ & Eur̆pium-1.5: & E曰. & 曻 & 200 \\
\hline $4400 \mathrm{~L}(E)$ & 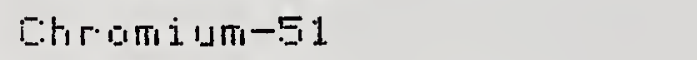 & 59 & 3 & $* \hbar$ \\
\hline $4400 H(E)$ & Etromium-5i. & 5 & 4 & \\
\hline
\end{tabular}


440JL(H) Iadire-131 Solutiar

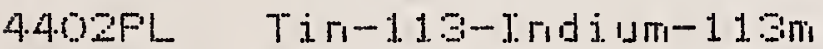

$4402 H(D)$ Tin-113-Iindium113m $501 \mathrm{n}$

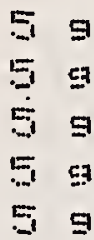

案 $* *$

4402L(E) Tin-L33-Jridium-11.3m 501r

$4403 H(E)$ Strantium-ES Salr

5

4403L(B) Strortjum-5E $501 \mathrm{r}$

59

4404H(E) THa11jum-201 E0ln

44OAL(E) ThET1 jum-201 $501 \mathrm{r}$

$4405 H(E)$ G01d-195 $501 \pi$

4.40L(E) G01d-198501n

59

5

5

\$ * * *

क $*$

$\$ * *$

\$

440GH(E) FHosfHorus-32 S01r

59

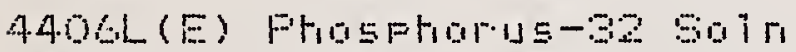

59

$4407 H(10)$ I gdine-125 Soln

$4407 \mathrm{~L}(\mathrm{Gi})$ Jodire-125 $501 \mathrm{r}$

4.0Bm Dabait-57.507r

5

59

59

$* *$

* $* *$

审 $*$

专 $* *$

中 $* *$

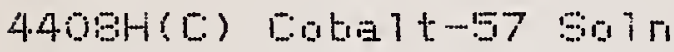

440GL(G) LobE]t-57501r

440\%H(I) $\Xi E 1$ enium-75 $501 \pi$

440 (D) (D) Selenium-75 501n

44.10H(H) Techretium-99m $501 \mathrm{r}$

59

59

59

59

59

क $*$

\$ $\#$

$\$ * *$

\$ $* *$

南

$4411 H(B)$ Techretium-g9m $501 \pi$

59

4.411L(E) ग.

4412H(G) Molrtadenum-99 $501 \pi$

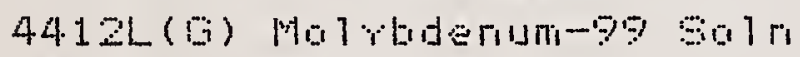

$4413 H \quad$ Ner Eurr-1975\%1r

5

5

59

59

\$ $* *$

क

中 $* *$

\$ $* *$

\$ $* *$

44.

$4414 \mathrm{H}(\mathrm{a})$ I adine-123 Hi Level Saln

5

59

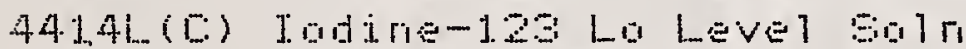

5

$4415 H(F) \quad x=n g n-133$ Dias

프

E.

$* *$

中 $* *$

क $* *$

$\$ * *$

韦 $*$

441SL(F) Xenon-13G lig

5

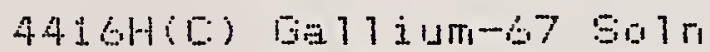

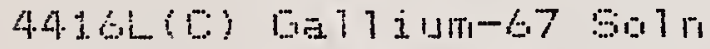

4.4.7H Indium-111 $501 \pi$

$4417 \mathrm{~L}$ Jndium-11. $501 \pi$

$44 \mathrm{sH}$ Mer.ur $-203501 \pi$

4.1.L Mer.urr-203517

$4419 H(E) \quad \gamma t t r i 1 \mathrm{H}-169501 \mathrm{n}$

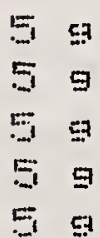

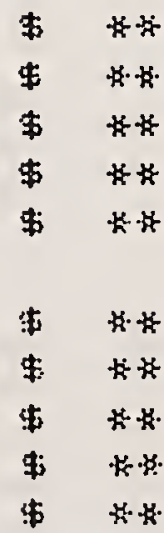

F FICE ANI AVAILABILITY DN FEDLEST: (301) $21-2605$ 


\begin{tabular}{|c|c|c|c|c|}
\hline $441 H$ & 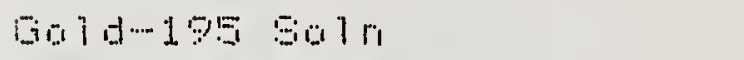 & 59 & w & $* 4$ \\
\hline 44.11 & 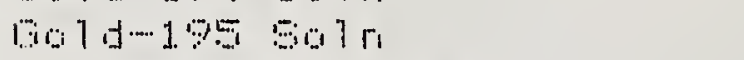 & 5 & \$ & $\hbar$ \\
\hline $4.42-1-1$ & rhlorins-36s01\% & 59 & \$ & ن \\
\hline $4422 \mathrm{~L}$ & rh 1 ar ine-go 501 & 55 & \$ & $*$ \\
\hline $404(F)$ & 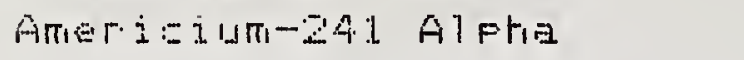 & $E \bar{z}$ & $\$$ & 97 \\
\hline $490(B)$ & 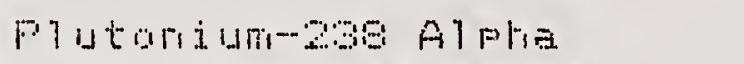 & 百司 & $\$$ & 1.69 \\
\hline $490 \%$ & 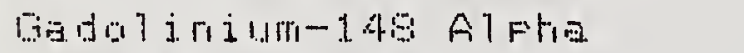 & ea & ; & 147 \\
\hline 41010$)$ & En & 59 & $\$$ & 246 \\
\hline $4019(5)$ & itrantiumang & $\because 9$ & \$ & $\forall *$ \\
\hline 49216$)$ & Gdim-2o Estat $501 \mathrm{r}$ & $\exists \leq$ & 宊 & $* *$ \\
\hline $4920(5)$ & $50 d i u n-2001 n$ & 둥 & ; & $x+x$ \\
\hline 14 & 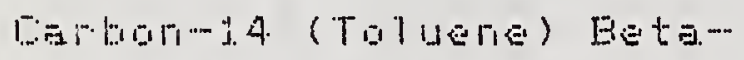 & $\because \pi i$ & $\$$ & *k \\
\hline 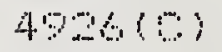 & 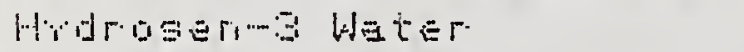 & 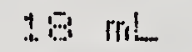 & 击 & 15 \\
\hline $497(\mathrm{E})$ & Wrdrogen-a Water. & $\because \quad$ riil & $\$$ & * \\
\hline $4200(0)$ & 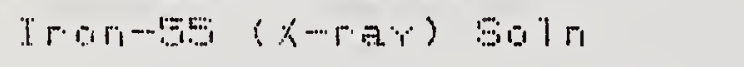 & $E \because$ & i & $*$ \\
\hline $490(E)$ & Hen & 5 & 犗 & $*$ \\
\hline ) & 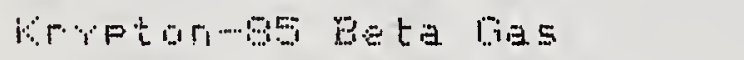 & 已ล & ; & 181 \\
\hline $4900(1)$ & Finothiun-1.47(Eeta) $501 \mathrm{~m}$ & 39 & 事 & 4 \\
\hline 4949 & 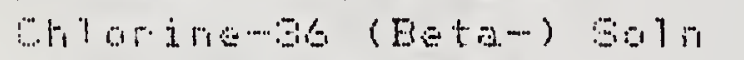 & $\Xi m i$ & $\$$ & 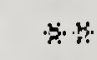 \\
\hline $4940: 0$ & 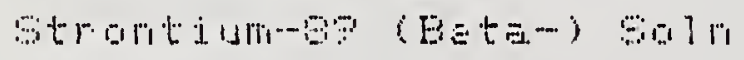 & $\because \mathrm{ril}$ & 虹 & $*$ \\
\hline $494 \%$ & 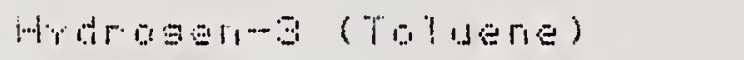 & 49 & 3 & 91 \\
\hline $474 \%(B)$ & 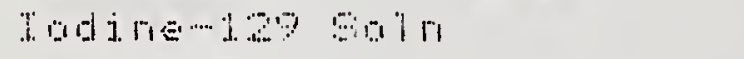 & 1. . 1. & 窟 & 394 \\
\hline $49010(1)$ & 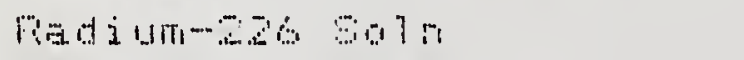 & 10,3 & \pm & 茨 \\
\hline 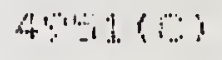 & MEd]. & $\mathrm{j} \cdot 4.4$ & sis & * k \\
\hline 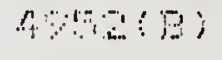 & Elarit: Golla lor Findon Amel & 209 & 苦 & 130 \\
\hline $40=0(5)$ & 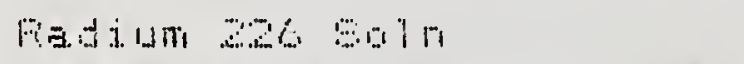 & 10.05 & 络 & i. 1. \\
\hline 40 & 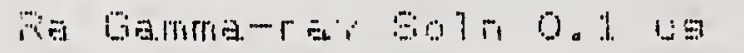 & $5 m_{1}$ & 苦 & +4 \\
\hline $4 \cdots$ & 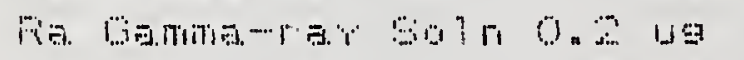 & $5.1 \quad \Leftrightarrow i$ & $\$$ & 1.74 \\
\hline 19 & 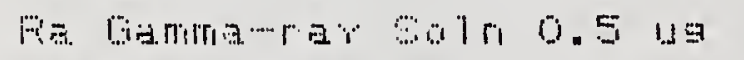 & 5.19 & 专 & 174 \\
\hline 45 & 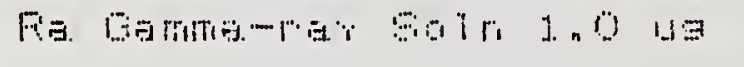 & : 1 & $\$$ & $\hbar *$ \\
\hline $4 \div$ & 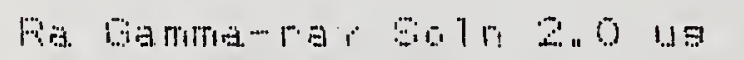 & $\therefore .1 \quad \exists$ & 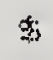 & 174 \\
\hline 400 & 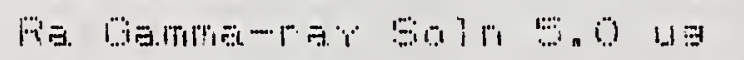 & $=1$ & t: & 14. \\
\hline 451 & 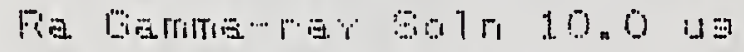 & ㄴ. $1 \quad 9$ & $\$$ & Kr: \\
\hline $4 \%$ & 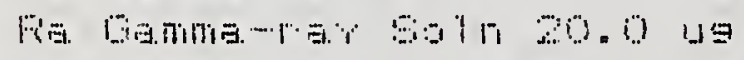 & w. & 密 & 1.74 \\
\hline 496 & Q & $\underline{1}, \mathrm{l} \quad \exists$ & 管 & 174 \\
\hline $4(4,6)$ & 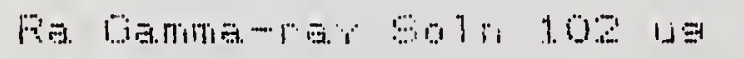 & 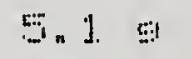 & : & 1.74 \\
\hline $40 \div(10)$ & $60+14 m_{i}-2$ & e & t; & 草 \\
\hline $490(\mathrm{E})$ & 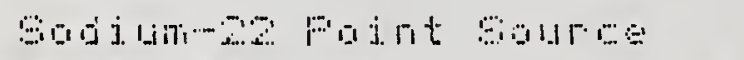 & $\Xi \Xi$ & s & 1.4 .6 \\
\hline 4976 & 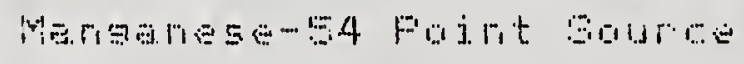 & a & $\hbar$ & 211 \\
\hline 4006$)$ & $\forall+t+j u m-6 \theta$ & ex & 密 & H. \\
\hline
\end{tabular}


$499 \%(F) \quad$ Cerium-139 Foint burre aro Magretio TeFe-Hi lieneitr

8000 NFL Meltjrg Faint bet

9001

NFL Garton EIt Sur Area.

8002

NFL Earton BIt: Sur Area

800

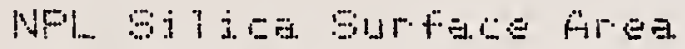

9004

8005

806

9007

NFL Bjlica Burfale Area

MFL Alumiria Surtage Area.

NFL Aiumira Burface Aré

NFL Alumina Gufare Area.

3008

NFl Alumira Surfare frea

3041

8042

9900

$0+6$

Nut ear Gortajuer sto gal.

NuElear Eortainer TrFe $A$

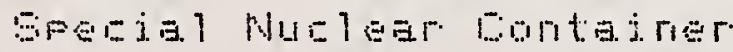

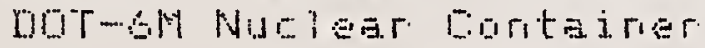

14794

IEA PUITEtrene IITA

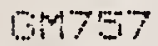

Div 78

iming:

IETA LUW TETTF RITA

IEYA HAd TENF ITA

Bim 760

rEP Mid Temp DTA

6) 761

nind

PNA

Fins:

Find?

IETA Hj.gi TEMF MTA

en

터.

set $(10)$

$10 \leqq$

$10 \exists$

109

109

505

509

50

+20

$\$ 1093$

क क

$\$$

落

$50 \quad 9$

e.

ea

servige

EA

结

古

+

ti

4

109

set $(5)$

set $(5)$

set (5)

est (5)

$\$ \quad$

$\$ 12$

安 25

$\$ 149$

226

Prot Altacore Tuna

TrA Thernogravjmetro

A1 Dute untra pujtr

Al Fod llitra Furity

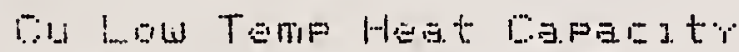

De⿻l一

set (5)

至五

a

도

259

事

$+96$

12

t 122

$\$ 122$

709

$100 \Rightarrow$

j. 드

19

1. 9

$* \quad 63$

$\$ 152$

$\$ \quad 9$

絭 79

$\$ 205$

11) 1002

onn Fiver Bedjurent

$11-\cdots 00$

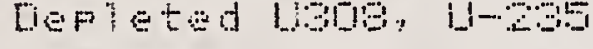

$11-010$

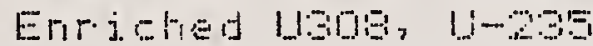

$16-0$

Enrictied L1909, L-205

$1-0,0$

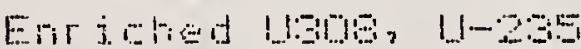

1.930

Brriched 1300, $1-2-35$

$(1-6)=0$

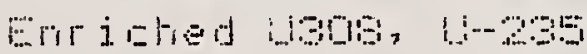

1,100

Enr iched 130, 11-235

19

1. 9

15

1. 9

19

$+86$

\$ 109

193

两 173

191

$1-150$

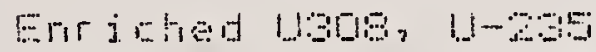

1. $\because$

\$ 191

क 191

क 179

\$1. 1.

$\$ 192$

11200

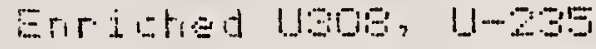

1 트

\$ 193

$11-300$

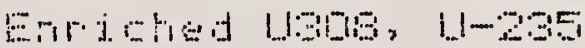

19

索 194

$1-.000$

Enr.itaed Hene, 11-205

$\$ 1.97$

19

走 199

$1,1-750$

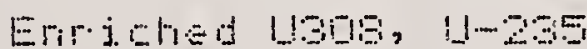

19

+30 24

* material in faefafiatign

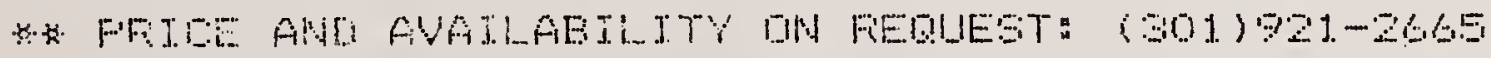




SEM

FFIIE

$11-800$

Enrighed 11:00:

$11-235$

1) 150

$1.1-900$

Enriched HEClE?

Enrighed HBLE,

Enriched lacio.

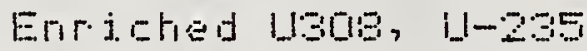

19

19

19

19

1.

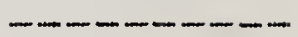

क 204

205

क 206

+ 209

$\$ 203$ 

